\title{
T-CONVERGENCE APPROACH TO VARIATIONAL PROBLEMS IN PERFORATED DOMAINS WITH FOURIER BOUNDARY CONDITIONS
}

\author{
VAleria Chiadò Piat ${ }^{1}$ And Andrey Piatnitski ${ }^{2}$
}

\begin{abstract}
The work focuses on the $\Gamma$-convergence problem and the convergence of minimizers for a functional defined in a periodic perforated medium and combining the bulk (volume distributed) energy and the surface energy distributed on the perforation boundary. It is assumed that the mean value of surface energy at each level set of test function is equal to zero. Under natural coercivity and $p$-growth assumptions on the bulk energy, and the assumption that the surface energy satisfies $p$-growth upper bound, we show that the studied functional has a nontrivial $\Gamma$-limit and the corresponding variational problem admits homogenization.
\end{abstract}

Mathematics Subject Classification. 35B27, 74Q05.

Received November 3rd, 2007. Revised June 24, 2008.

Published online December 19, 2008.

\section{INTRODUCTION}

This work is devoted to the asymptotic analysis of a variational problem for a functional defined in a perforated medium and combining the bulk (volume distributed) energy and the surface energy defined on the perforation boundary. In the studied model the perforation is obtained by a homothetic dilatation of a given periodic structure of holes, with a small scaling factor denoted by $\varepsilon$. Then the surface measure tends to infinity as $\varepsilon$ goes to 0 . To compensate this measure growth we assume that the mean value of surface energy at each level set of the unknown function is equal to zero. Then, under proper coercivity assumptions on the bulk energy, we show that the said functional has a nontrivial $\Gamma$-limit and the corresponding variational problem is well-posed and admits homogenization.

The behaviour of solutions to boundary value problems in perforated domains with Neumann boundary condition at the microstructure boundary is well understood now. There is an extensive literature on this subject. We refer here the works [9,13], where both Neumann and Dirichlet boundary conditions were considered. The paper [11] dealt with the Stokes and Navier-Stokes equations in perforated domains. In the work [6] the variational approach was used to study boundary value problems for Poisson equation in perforated domain.

The linear elliptic equations in perforated domain with Dirichlet and Fourier boundary conditions on the boundary of the perforation were considered in several mathematical works. The case of Dirichlet problem in a periodic perforated medium was investigated in $[8,13]$. It was shown that, if the volume fraction of the perforation does not vanish, then the solution vanishes at the rate $\varepsilon^{2}$. If the volume fraction of the perforation

\footnotetext{
Keywords and phrases. Homogenization, $\Gamma$-convergence, perforated medium.

1 Dipartimento di Matematica, Politecnico di Torino, C.so Duca degli Abruzzi 24, 10129 Torino, Italy.

2 Narvik University College, HiN, Postbox 385, 8505, Narvik, Norway and Lebedev Physical Institute RAS, Leninski prospect 53,

Moscow 119991, Russia. andrey@sci.lebedev.ru 
is asymptotically small, then, under a proper choice of the rate of its decay, the homogenized equation might receive an additional potential (the so called "strange term"). This phenomenon was observed in $[8,13,14]$. The problem with dissipative Fourier condition on the boundary of the perforation was considered in $[7,10]$, and some other works. In the case of homothetic dilatation of a given periodic perforated structure, the solution vanishes, as the microstructure period tends to zero. However, if the coefficient of the Fourier boundary operator is small (of order $\varepsilon$ ), or the volume fraction of the holes vanishes at a certain critical rate, then the homogenization result holds, and the limit operator has an additional potential (see $[3,15,16,18])$.

The case of Fourier boundary condition with the coefficient having zero average over the perforation surface, has been considered in [4]; closely related spectral problems have also been studied in [17,19]. In this case the formally homogenized operator is well-defined and contains an additional potential. If this homogenized operator is coercive, then the original problem is well-posed for all sufficiently small $\varepsilon$ and the studied family of problems admits homogenization. This is a linear version of the problem studied in the present paper, the corresponding Lagrangian in this case being purely quadratic.

$\Gamma$-convergence and homogenization of variational functionals with periodic and locally periodic Lagrangians have been widely studied in the existing literature, see for instance $[5,12]$.

In the model studied in this work, the bulk energy density (denoted by $f\left(\frac{x}{\varepsilon}, D u\right)$ ) is periodic in the space variable and satisfies convexity, coercivity and $p$-growth conditions with respect to the gradient of the unknown function. The surface energy density (denoted by $g\left(\frac{x}{\varepsilon}, u(x)\right)$ ) is a periodic function of the first argument, which admits a $p$-growth upper bound and satisfies a local Lipschitz condition with respect to $u$. We assume that the mean value of $g(\cdot, z)$ over the perforation surface is equal to zero for any $z \in \mathbb{R}$. This condition is crucial.

We show that under mentioned above conditions the studied functional $\Gamma$-converges to the limit functional defined in the solid domain. The limit Lagrangian is determined in terms of an auxiliary variational problem on the perforated torus. It is worth to note that, in contrast with the linear case mentioned above, the contributions of the bulk and surface energies to the limit Lagrangian are coupled.

We then prove that, if the coerciveness constant of the bulk energy is large enough, then the functional under consideration is coercive uniformly in $\varepsilon$. This allows us to study the asymptotic behaviour of the corresponding minimization problems and show that the minimal energies and minimizers of the $\varepsilon$-variational problems converge to those of the limit functional.

The paper is organized as follows:

Section 1 contains the problem setup and main definitions. Then, in Section 2 we introduce sufficient conditions for the coercivity of the studied energy functionals, and state our main results. In Section 3 we prove some auxiliary statements. Sections 4 and 5 deal with the proof of $\Gamma$-liminf and $\Gamma$-limsup inequalities respectively.

\section{Assumptions And Setting of the Problem}

Let $Y=[0,1)^{n}$. Let also $E \subset \mathbb{R}^{n}$ be a $Y$-periodic, connected, open set, with Lipschitz boundary $\partial E=S$, and denote $B=\mathbb{R}^{n} \backslash E$. We also assume for the presentation simplicity that $E \cap Y$ is a connected set with Lipschitz boundary and that $B \cap Y \subset \subset Y$ so that $\mathbb{R}^{n} \backslash E$ is made of disconnected components. Denote $E_{0}=E \cap Y$, $S_{0}=S \cap Y, B_{0}=B \cap Y$.

For any positive number $\varepsilon$ and every set $A \subset \mathbb{R}^{n}$ we denote the corresponding $\varepsilon$-homothetic set by $\varepsilon A=\left\{x \in \mathbb{R}^{n}: x / \varepsilon \in A\right\}$. Now, for every $i \in \mathbb{Z}^{n}$ we set $Y_{\varepsilon}^{i}=\varepsilon(i+Y), S_{\varepsilon}^{i}=\varepsilon S \cap Y_{\varepsilon}^{i}, B_{\varepsilon}^{i}=\varepsilon B \cap Y_{\varepsilon}^{i}$. Given a bounded open set $\Omega \subset \mathbb{R}^{n}$ with Lipschitz boundary, we consider the perforated domain $\Omega_{\varepsilon}$ defined by

$$
\Omega_{\varepsilon}=\Omega \backslash \cup\left\{B_{\varepsilon}^{i}: i \in I_{\varepsilon}\right\}, \quad I_{\varepsilon}=\left\{i \in \mathbb{Z}^{n}: Y_{\varepsilon}^{i} \subset \Omega\right\} .
$$

In this case $\Omega_{\varepsilon}$ remains connected and the perforation does not intersect the boundary of $\Omega$, so that $\partial \Omega_{\varepsilon}$ is the union of the fixed surface $\partial \Omega$ and the varying surface $S_{\varepsilon}$

$$
\partial \Omega_{\varepsilon}=\partial \Omega \cup S_{\varepsilon}, \quad S_{\varepsilon}=\cup\left\{S_{\varepsilon}^{i}: i \in I_{\varepsilon}\right\}
$$


Let us denote by $\mathcal{H}^{n-1}$ the Hausdorff $(n-1)$-dimensional measure in $\mathbb{R}^{n}$, and let $\overline{\mathbb{R}}=\mathbb{R} \cup\{-\infty,+\infty\}$. We consider the functional $F_{\varepsilon}: L^{p}(\Omega) \rightarrow \overline{\mathbb{R}}$ defined by

$$
F_{\varepsilon}(u)= \begin{cases}\int_{\Omega_{\varepsilon}} f\left(\frac{x}{\varepsilon}, D u\right) \mathrm{d} x+\int_{S_{\varepsilon}} g\left(\frac{x}{\varepsilon}, u\right) \mathrm{d} \mathcal{H}^{n-1} & \text { if } u \in W^{1, p}\left(\Omega_{\varepsilon}\right) \\ +\infty & \text { otherwise. }\end{cases}
$$

Here $f=f(y, \xi): \mathbb{R}^{n} \times \mathbb{R}^{n} \rightarrow \mathbb{R}, g=g(y, z): \mathbb{R}^{n} \times \mathbb{R} \rightarrow \mathbb{R}$ are given Borel functions, which satisfy the following conditions:

- $f(y, \xi)$ and $g(y, z)$ are $Y$-periodic in variable $y$.

- $f(y, \xi)$ is convex in $\xi$.

- $p$-growth: there is $p>1$ such that

$$
\begin{gathered}
c_{1}|\xi|^{p} \leq f(y, \xi) \leq c_{2}\left(1+|\xi|^{p}\right) \\
|g(y, z)| \leq c_{3}\left(1+|z|^{p}\right), \quad\left|g_{z}^{\prime}(y, z)\right| \leq c_{3}\left(1+|z|^{p-1}\right)
\end{gathered}
$$

for almost all $y \in \mathbb{R}^{n}$, all $\xi \in$ and $z \in \mathbb{R}$.

- Centering:

- Lipschitz continuity:

$$
\int_{S} g(y, z) \mathrm{d} \mathcal{H}^{n-1}(y)=0 \quad \text { for all } z \in \mathbb{R} .
$$

$$
\begin{gathered}
\left|g\left(y, z_{1}\right)-g\left(y, z_{2}\right)\right| \leq c_{4}\left(1+\left|z_{1}\right|+\left|z_{2}\right|\right)^{p-1}\left|z_{1}-z_{2}\right| \\
\left|g_{z}^{\prime}\left(y, z_{1}\right)-g_{z}^{\prime}\left(y, z_{2}\right)\right| \leq c_{5}\left(1+\left|z_{1}\right|+\left|z_{2}\right|\right)^{p-2}\left|z_{1}-z_{2}\right|
\end{gathered}
$$

for all $z_{1}, z_{2} \in \mathbb{R}$.

Actually, (1.6) is a consequence of (1.4). We formulate this condition explicitly for the sake of convenience. Also notice that, due to the convexity of $f(y, \cdot),(1.3)$ implies the estimate

$$
\left|f\left(y, \xi_{1}\right)-f\left(y, \xi_{2}\right)\right| \leq c_{6}\left(1+\left|\xi_{1}\right|+\left|\xi_{2}\right|\right)^{p-1}\left|\xi_{1}-\xi_{2}\right| .
$$

Given $\Phi \in W_{\text {loc }}^{1, p}\left(\mathbb{R}^{n}\right)$, we consider a minimization problem with Dirichlet boundary conditions on the exterior boundary $\partial \Omega$, namely

$$
m_{\varepsilon}=\min \left\{F_{\varepsilon}(u): u=\Phi \text { on } \partial \Omega\right\}
$$

and study the asymptotic behaviour of $m_{\varepsilon}$ and the corresponding minimizers as $\varepsilon \rightarrow 0$. We remark that the surface integral in the functional (1.2) plays the role of a boundary condition of Fourier-type on the varying part of $\partial \Omega_{\varepsilon}$.

Notice that the minimizers of $F_{\varepsilon}$ are only defined in $\Omega_{\varepsilon}$. It is convenient to extend them to the whole domain $\Omega$.

Lemma 1.1. Under our standing assumptions on the geometry of $\Omega_{\varepsilon}$ there exists a family of linear continuous extension operators

$$
T_{\varepsilon}: W^{1, p}\left(\Omega_{\varepsilon}\right) \rightarrow W^{1, p}(\Omega)
$$

such that

$$
T_{\varepsilon} u=u \text { in } \Omega_{\varepsilon}
$$

and

$$
\int_{\Omega}\left|T_{\varepsilon} u\right|^{p} \mathrm{~d} x \leq C \int_{\Omega_{\varepsilon}}|u|^{p} \mathrm{~d} x, \quad \int_{\Omega}\left|D\left(T_{\varepsilon} u\right)\right|^{p} \mathrm{~d} x \leq C \int_{\Omega_{\varepsilon}}|D u|^{p} \mathrm{~d} x
$$

for each $u \in W^{1, p}\left(\Omega_{\varepsilon}\right)$, the constant $C>0$ here does not depend on $\varepsilon$. 
Proof. In the case $p=2$ the proof of the required statement can be found in [9] and in [10], Theorem 1.2.10. Following the line of this proof, one can easily show that this statement also holds for any $p \in(1,+\infty)$. Indeed, for an arbitrary function $u \in W^{1, p}\left(\Omega_{\varepsilon}\right)$, denote by $u_{\varepsilon}^{i}, i \in I_{\varepsilon}$, the restriction of $u$ on $Y_{\varepsilon}^{i}$, and by $U_{\varepsilon}^{i}$ the rescaled functions

$$
U_{\varepsilon}^{i}(y)=u_{\varepsilon}^{i}(\varepsilon(y+i)) .
$$

By construction, $U_{\varepsilon}^{i} \in W^{1, p}\left(Y \backslash B_{0}\right)$. Since, under our assumptions, $E_{0}=Y \cap E$ is a connected set with a Lipschitz boundary, then, according to Lemma 2.6 in [1], there exists an extension operator $T: W^{1, p}\left(Y \backslash B_{0}\right) \rightarrow W^{1, p}(Y)$ such that for any $U \in W^{1, p}\left(Y \backslash B_{0}\right)$ it holds $T U=U$ in $Y \backslash B_{0}$, and

$$
\int_{Y}|T U|^{p} \mathrm{~d} y \leq C \int_{Y \backslash B_{0}}|U|^{p} \mathrm{~d} y, \quad \int_{Y}|D(T U)|^{p} \mathrm{~d} y \leq C \int_{Y \backslash B_{0}}|D U|^{p} \mathrm{~d} y .
$$

The proof of quoted above Lemma 2.6 in [1] is based on subtracting from $U$ its mean value and applying the Poincaré inequality to the obtained function (see [1] for the details). If we denote the extension $T U_{\varepsilon}^{i}$ by $\tilde{U}_{\varepsilon}^{i}$ and let $\tilde{u}_{\varepsilon}^{i}(x)=\tilde{U}_{\varepsilon}^{i}\left(\frac{x-i}{\varepsilon}\right)$, then $\tilde{u}_{\varepsilon}^{i}=u$ in $Y_{\varepsilon}^{i} \backslash B_{\varepsilon}^{i}$, and

$$
\int_{Y_{\varepsilon}^{i}}\left|\tilde{u}_{\varepsilon}^{i}\right|^{p} \mathrm{~d} x \leq C \int_{Y_{\varepsilon}^{i} \backslash B_{\varepsilon}^{i}}|u|^{p} \mathrm{~d} x, \quad \int_{Y_{\varepsilon}^{i}}\left|D \tilde{u}_{\varepsilon}^{i}\right|^{p} \mathrm{~d} x \leq C \int_{Y_{\varepsilon}^{i} \backslash B_{\varepsilon}^{i}}|D u|^{p} \mathrm{~d} x
$$

for all $i \in I_{\varepsilon}$. Setting $T_{\varepsilon} u=u_{\varepsilon}^{i}$ for $x \in Y_{\varepsilon}^{i}$ and $T_{\varepsilon} u=u$ for $x \in \Omega \backslash \bigcup\left\{Y_{\varepsilon}^{i}: i \in I_{\varepsilon}\right\}$, we obtain the desired extension operator $T_{\varepsilon}$. The estimate (1.10) can be obtained by summing up the inequalities (1.11) over $i \in I_{\varepsilon}$. This completes the proof.

For the notation simplicity, in this paper we will keep the notation $u$ also for the extended function $T_{\varepsilon} u$.

As a consequence of the existence of extension operators one can derive Friedrichs inequality: there exists a constant $k_{f}>0$ depending only on $p, n, \Omega$, such that

$$
\int_{\Omega_{\varepsilon}}|u|^{p} \mathrm{~d} x \leq k_{f} \int_{\Omega_{\varepsilon}}|D u|^{p} \mathrm{~d} x
$$

for all $\varepsilon>0$ and all $u \in W^{1, p}\left(\Omega_{\varepsilon}\right)$, such that $u=0$ on $\partial \Omega$.

Notice that the functional (1.2) need not be equi-coercive in $L^{p}(\Omega)$, and the infimum in (1.9) might be equal to $-\infty$. In this case the boundedness of the energies $F_{\varepsilon}(u)$ does not imply any a priori estimates for $u$. The corresponding example with quadratic functions $f(x, \cdot), g(x, \cdot)$ can be constructed as follows.

Let $\Omega=(0,1)^{n}$ be a unit cube in $\mathbb{R}^{n}$, and suppose that $B$ is a $[0,1]^{n}$-periodic cubic structure in $\mathbb{R}^{n}$, generated by the set $B_{0}=[r, 1-r]^{n}$ with $r=1 / 4$. Then $\varepsilon B$ is a disperse cubic structure. Denote $S_{\varepsilon}=\varepsilon \partial B \cap \Omega$. We consider the functional

$$
F_{\varepsilon}(u)=\int_{\Omega_{\varepsilon}}|\nabla u|^{2} \mathrm{~d} x+\lambda \int_{S_{\varepsilon}} g_{0}\left(\frac{x}{\varepsilon}\right) u^{2} \mathrm{~d} \mathcal{H}^{n-1}, \quad \text { with } \lambda>0
$$

and we assume that $g_{0}(y)$ is a smooth $Y$-periodic function whose trace on $S$ is nontrivial (not equal to 0 ) and satisfies the condition

$$
\int_{S_{0}} g_{0}(y) \mathrm{d} \mathcal{H}^{n-1}=0 .
$$

This is a particular case of $(1.2)$ with $f(y, z, \xi)=|\xi|^{2}$ and $g(y, z)=g_{0}(y) z^{2}$. Clearly, all the conditions (1.3)-(1.8) are fulfilled with $p=2$. Denote

$$
u_{0}(x)=\left[x_{1}\left(1-x_{1}\right)\right]^{2}\left[x_{2}\left(1-x_{2}\right)\right]^{2} \times \ldots \times\left[x_{n}\left(1-x_{n}\right)\right]^{2}
$$


and

$$
u_{\varepsilon}(x)=u_{0}(x)-\varepsilon g_{0}\left(\frac{x}{\varepsilon}\right) u_{0}(x) .
$$

In this case $u_{\varepsilon}=u_{0}=\Phi$ on $\partial \Omega$, with $\Phi \equiv 0$. Evaluating $F_{\varepsilon}\left(u_{\varepsilon}\right)$ we obtain after straightforward rearrangements

$$
\begin{aligned}
F_{\varepsilon}\left(u_{\varepsilon}\right)= & \int_{E_{0}} \mathrm{~d} y \int_{\Omega}\left|\nabla u_{0}(x)\right|^{2} \mathrm{~d} x+\int_{\Omega} u_{0}^{2}(x) \int_{E_{0}}\left|\nabla g_{0}(y)\right|^{2} \mathrm{~d} y \mathrm{~d} x \\
& +\lambda \int_{S_{\varepsilon}} u_{0}^{2}(x) g_{0}\left(\frac{x}{\varepsilon}\right) \mathrm{d} \mathcal{H}^{n-1}-2 \varepsilon \lambda \int_{S_{\varepsilon}}\left|u_{0}(x)\right|^{2} g_{0}^{2}\left(\frac{x}{\varepsilon}\right) \mathrm{d} \mathcal{H}^{n-1}+O(\varepsilon) \\
= & \left(1-(1-2 r)^{n}\right) \int_{\Omega}\left|\nabla u_{0}(x)\right|^{2} \mathrm{~d} x+\int_{\Omega} u_{0}^{2}(x) \int_{E_{0}}\left|\nabla g_{0}(y)\right|^{2} \mathrm{~d} y \mathrm{~d} x \\
& +2 \lambda \int_{\Omega} \nabla\left(u_{0}^{2}(x)\right) \mathrm{d} x \cdot \int_{S_{0}} y g_{0}(y) \mathrm{d} \mathcal{H}^{n-1}(y)-2 \lambda \int_{\Omega} \int_{S_{0}} u_{0}^{2}(x) g_{0}^{2}(y) \mathrm{d} \mathcal{H}^{n-1}(y) \mathrm{d} x+O(\varepsilon) .
\end{aligned}
$$

If we now choose $g_{0}(y)$ in such a way that

- $g_{0}(y)$ only depends on $y_{1}$, i.e. $g_{0}(y)=g_{0}\left(y_{1}\right)$;

- $g_{0}\left(y_{1}-1 / 2\right)$ is an even function;

$-\operatorname{supp}\left(g_{0}(\cdot)\right) \cap[0,1] \subset(-1 / 4,1 / 4)$,

$-\int_{0}^{1} g_{0}\left(y_{1}\right) \mathrm{d} y_{1}=0$,

then the third integral on the r.h.s. of the last formula is equal to zero, and we get

$$
\begin{gathered}
F_{\varepsilon}\left(u_{\varepsilon}\right)=\left(1-(1 / 4)^{n}\right) \int_{\Omega}\left|\nabla u_{0}(x)\right|^{2} \mathrm{~d} x+\int_{\Omega} u_{0}^{2}(x) \int_{E_{0}}\left|\nabla g_{0}(y)\right|^{2} \mathrm{~d} y \mathrm{~d} x \\
-2 \lambda \int_{\Omega} \int_{S_{0}} u_{0}^{2}(x) g_{0}^{2}(y) \mathrm{d} \mathcal{H}^{n-1}(y) \mathrm{d} x+O(\varepsilon) .
\end{gathered}
$$

Since $\int_{\Omega} u_{0}^{2}(x) \mathrm{d} x>0$ and $\int_{S_{0}} g_{0}^{2}(y) \mathrm{d} \mathcal{H}^{n-1}(y)>0$, then for large enough $\lambda$ and small enough $\varepsilon$ we obtain $F_{\varepsilon}\left(u_{\varepsilon}\right) \leq c<0$. Therefore, if we denote $v_{\varepsilon}(x)=\varepsilon^{-1} u_{\varepsilon}(x)$, then, for some $\lambda>0$,

$$
F_{\varepsilon}\left(v_{\varepsilon}\right)=\varepsilon^{-2} F_{\varepsilon}\left(u_{\varepsilon}\right) \leq c \varepsilon^{-2}<0, \quad \text { and } \quad\left\|v_{\varepsilon}\right\|_{L^{2}(\Omega)} \rightarrow \infty, \text { as } \varepsilon \rightarrow 0 .
$$

In this paper we will show that the functional $F_{\varepsilon}$ does $\Gamma$-converge, as $\varepsilon \rightarrow 0$ (see, for instance [12] for the definition of $\Gamma$-convergence), and that the limit functional $F$ takes the form

$$
F(u)= \begin{cases}\int_{\Omega} L(u, D u) \mathrm{d} x & \text { if } u \in W^{1, p}(\Omega) \\ +\infty & \text { otherwise }\end{cases}
$$

where

$$
L(z, \xi)=\inf \left\{\int_{E \cap Y} f(y, \xi+D w) \mathrm{d} y+\int_{S \cap Y} g_{z}^{\prime}(y, z)(\xi \cdot y+w) \mathrm{d} \mathcal{H}^{n-1}: w \in W_{\text {per }}^{1, p}(Y \cap E)\right\} .
$$


Here $W_{\text {per }}^{1, p}(E \cap Y)$ denotes the space of functions $w: E \rightarrow \mathbb{R}$ being the restriction to $E$ of $Y$-periodic functions in $W_{\text {loc }}^{1, p}\left(\mathbb{R}^{n}\right)$.

We also show that, under the assumption that the coercivity constant $c_{1}$ in (1.3) is sufficiently large, the minimizer $u_{\varepsilon}$ in (1.9) converges in $L^{p}(\Omega)$, as $\varepsilon \rightarrow 0$, towards a minimizer $u$ of the limit functional $F$. Moreover, the corresponding minimum values also converge, i.e., $m_{\varepsilon} \rightarrow m$ where

$$
m=\min \{F(u): u=\Phi \text { on } \partial \Omega\}
$$

Let us compute the effective Lagrangian in the quadratic case:

$$
f(y, \xi)=a(y) \xi \cdot \xi, \quad g(y, z)=g_{0}(y) z^{2} .
$$

According to the above formula (1.14), in this case we have

$$
L(z, \xi)=\min _{w \in H_{\mathrm{per}}^{1}(Y \cap E)}\left\{\int_{E \cap Y} a(y)(\xi+D w) \cdot(\xi+D w) \mathrm{d} y+2 \int_{S \cap Y} g_{0}(y) z(\xi \cdot y+w) \mathrm{d} \mathcal{H}^{n-1}\right\}
$$

The corresponding Euler equation reads

$$
\begin{cases}\operatorname{div}(a(y)(\xi+\nabla w(y)))=0 & \text { in } E_{0} \\ \left.\frac{\partial}{\partial n_{a}} w\right|_{S_{0}}=-a(y) n \cdot \xi+z g_{0}(y), & w \in H_{\mathrm{per}}^{1}(Y)\end{cases}
$$

By linearity, a solution of this equation can be represented as the sum $w(x)=w_{1}(x)+w_{2}(x)$, where $w_{1}$ and $w_{2}$ are solutions to the problems

$$
\begin{cases}\operatorname{div}\left(a(y)\left(\xi+\nabla w_{1}(y)\right)\right)=0 & \text { in } E_{0} \\ \left.\frac{\partial}{\partial n_{a}} w_{1}\right|_{S_{0}}=-a(y) n \cdot \xi, & w_{1} \in H_{\mathrm{per}}^{1}(Y)\end{cases}
$$

and

$$
\begin{cases}\operatorname{div}\left(a(y) \nabla w_{2}(y)\right)=0 & \text { in } E_{0} \\ \left.\frac{\partial}{\partial n_{a}} w_{2}\right|_{S_{0}}=z g_{0}(y) & w_{2} \in H_{\mathrm{per}}^{1}(Y) .\end{cases}
$$

Substituting $w_{1}$ and $w_{2}$ in (1.16) and considering the above equations and the fact that $w_{1}$ and $w_{2}$ depend linearly on $\xi$ and $z$ respectively, we obtain after simple rearrangements

$$
\begin{aligned}
L(z, \xi)= & \int_{E_{0}} a(y)\left(\xi+\nabla w_{1}\right) \cdot\left(\xi+\nabla w_{1}\right) \mathrm{d} y-\int_{E_{0}} a(y) \nabla w_{2} \cdot \nabla w_{2} \mathrm{~d} y \\
& +2 \int_{S_{0}} g_{0}(y) z\left(\xi \cdot y+w_{1}(y)\right) \mathrm{d} \mathcal{H}^{n-1}=\hat{a}^{N} \xi \cdot \xi-\hat{g} z^{2}+\hat{b} \cdot \xi z .
\end{aligned}
$$

It should be noted that the matrix $\hat{a}^{N}$ here coincides with the effective matrix for the classical homogenization problem with homogeneous Neumann conditions on the perforation boundary.

Notice also that $\hat{g}>0$ unless $g_{0}(y)=0$. The contribution of the last term $\hat{b} \cdot \xi z$ can be computed explicitly. Indeed, if $u=\Phi$ on $\partial \Omega$, then

$$
\frac{1}{2} \int_{\Omega} \hat{b} \cdot \nabla\left(u^{2}(x)\right) \mathrm{d} x=\frac{1}{2} \int_{\partial \Omega} \hat{b} \cdot n \Phi^{2}(x) \mathrm{d} \mathcal{H}^{n-1} .
$$

Therefore, this term does not depend on $u$, and hence it does not have an influence on the limit minimizer. 


\section{Main Results}

First of all, we obtain the estimate for the surface term of $F_{\varepsilon}(u)$ in terms of its volume term and the $L^{p}$-norm of $u$ on $\Omega_{\varepsilon}$. This estimate relies crucially on the assumption (1.5) and plays important role in the further analysis.

Lemma 2.1. For any $\gamma>0$ there exists a positive constant $c(\gamma)$ such that for each $\varepsilon>0$ and every set $A_{\varepsilon}$ of the form

the inequality holds

$$
A_{\varepsilon}=\cup\left\{Y_{\varepsilon}^{i} \cap(\varepsilon E): i \in I\right\}, \quad \text { with } I \subset I_{\varepsilon}
$$

$$
\left|\int_{S_{\varepsilon} \cap A_{\varepsilon}} g\left(\frac{x}{\varepsilon}, u\right) \mathrm{d} \mathcal{H}^{n-1}\right| \leq\left(\gamma+\varepsilon^{p} c(\gamma)\right) \int_{A_{\varepsilon}}|D u|^{p} \mathrm{~d} x+c(\gamma) \int_{A_{\varepsilon}}\left(1+|u|^{p}\right) \mathrm{d} x
$$

for all $u \in W^{1, p}\left(A_{\varepsilon}\right)$. Moreover,

$$
\left|\int_{S_{\varepsilon}} g\left(\frac{x}{\varepsilon}, u\right) \mathrm{d} \mathcal{H}^{n-1}\right| \leq\left(\gamma+\varepsilon^{p} c(\gamma)\right) \int_{\Omega_{\varepsilon}}|D u|^{p} \mathrm{~d} x+c(\gamma) \int_{\Omega_{\varepsilon}}\left(1+|u|^{p}\right) \mathrm{d} x
$$

for all $u \in W^{1, p}\left(\Omega_{\varepsilon}\right)$. In particular,

$$
\left|\int_{S_{\varepsilon}} g\left(\frac{x}{\varepsilon}, u\right) \mathrm{d} \mathcal{H}^{n-1}\right| \leq k_{0} \int_{\Omega_{\varepsilon}}\left(1+|u|^{p}+|D u|^{p}\right) \mathrm{d} x
$$

for some $k_{0}>0$ which does not depend on $\varepsilon$.

The proof of Lemma 2.1 is given in Section 3.

For the reader's convenience, we recall now the definition of $\Gamma$-convergence that we will use in the rest of the paper.

Definition 2.2. Let $F_{\varepsilon}, F: L^{p}(\Omega) \rightarrow \overline{\mathbb{R}}$ for every $\varepsilon>0$. The family $F_{\varepsilon}$ is said to $\Gamma$-converge to $F$, as $\varepsilon \rightarrow 0$, if the following two properties hold

(a) ( $\Gamma$-lim inf inequality) For any sequence $u_{\varepsilon} \in L^{p}(\Omega)$, such that $u_{\varepsilon}$ converges to $u$ in $L^{p}(\Omega)$, as $\varepsilon \rightarrow 0$, we have

$$
\liminf _{\varepsilon \rightarrow 0} F_{\varepsilon}\left(u_{\varepsilon}\right) \geq F(u)
$$

(b) ( $\Gamma$-limsup inequality) For any $u \in L^{p}(\Omega)$ there is a sequence $u_{\varepsilon} \in L^{p}(\Omega)$ such that $u_{\varepsilon}$ converges to $u$ in $L^{p}(\Omega)$ and

$$
\limsup _{\varepsilon \rightarrow 0} F_{\varepsilon}\left(u_{\varepsilon}\right) \leq F(u)
$$

We state now our main result.

Theorem 2.3. Let $F_{\varepsilon}, F: L^{p}(\Omega) \rightarrow \overline{\mathbb{R}}$ be the functional given by (1.2), (1.13), (1.14), and suppose that all the conditions specified in Section 1 are fulfilled. Then $F_{\varepsilon}$ does $\Gamma$-converge to $F$, as $\varepsilon \rightarrow 0$.

Proposition 2.4. Let $u \in W^{1, p}(\Omega)$, and suppose that $\left.u\right|_{\partial \Omega}=\Phi$. Then there is a family $u_{\varepsilon} \in W^{1, p}(\Omega)$, $\left.u_{\varepsilon}\right|_{\partial \Omega}=\Phi$, such that

$$
\limsup _{\varepsilon \rightarrow 0} F_{\varepsilon}\left(u_{\varepsilon}\right)=F(u)
$$

The proof of Theorem 2.3 and Proposition 2.4 is presented in the following sections. In the rest of this section we derive a number of consequences of these results. Consider minimization problems (1.9) and (1.15). 
Corollary 2.5. If all the assumptions of Theorem 2.3 hold true and if, furthermore,

$$
c_{1}>k_{0}\left(1+k_{f}\right)
$$

where $k_{f}$ is given by (1.12), then for every $\Phi \in W_{\mathrm{loc}}^{1, p}\left(\mathbb{R}^{n}\right)$ and for all sufficiently small $\varepsilon>0$ problem (1.9) is well-posed and has a minimizer $u_{\varepsilon} \in W^{1, p}(\Omega)$. Moreover, the limit problem (1.15) is also well-posed, and

$$
\int_{\Omega_{\varepsilon}}\left|u_{\varepsilon}-u\right|^{p} \mathrm{~d} x \rightarrow 0, \quad m_{\varepsilon} \rightarrow m
$$

as $\varepsilon \rightarrow 0$, where $u$ is a solution to problem (1.15), and $m$ is the corresponding minimum.

Proof. Let us first show that under condition (2.5) the functionals $\left\{F_{\varepsilon}(u):\left.u\right|_{\partial \Omega}=\Phi\right\}$ are equi-coercive. For any $u \in W^{1, p}\left(\Omega_{\varepsilon}\right)$ by $(1.3)$ and $(2.3)$ we have

$$
F_{\varepsilon}(u) \geq-k_{0} \int_{\Omega_{\varepsilon}}\left(1+|u|^{p}+|D u|^{p}\right) \mathrm{d} x+c_{1} \int_{\Omega_{\varepsilon}}|D u|^{p} \mathrm{~d} x
$$

Since $\left.u\right|_{\partial \Omega}=\Phi,(1.12)$ we get

$$
\int_{\Omega_{\varepsilon}}|u-\Phi|^{p} \mathrm{~d} x \leq k_{f} \int_{\Omega_{\varepsilon}}|D u-D \Phi|^{p} \mathrm{~d} x
$$

We transform this estimate using the following simple inequality: for any $\kappa>0$ there is $c(p, \kappa)>0$ such that $(a+b)^{p} \leq(1+\kappa) a^{p}+c(p, \kappa) b^{p}$ for all positive $a$ and $b$. After simple rearrangements this yields

$$
\int_{\Omega_{\varepsilon}}|u|^{p} \mathrm{~d} x \leq(1+\kappa) k_{f} \int_{\Omega_{\varepsilon}}|D u|^{p} \mathrm{~d} x+c(p, \kappa)\left(1+k_{f}\right) \int_{\Omega_{\varepsilon}}\left(|\Phi|^{p}+|D \Phi|^{p}\right) \mathrm{d} x .
$$

Combining the last estimate with (2.7), we obtain

$$
\left(c_{1}-k_{0}\left(1+(1+\kappa) k_{f}\right)\right) \int_{\Omega_{\varepsilon}}|D u|^{p} \mathrm{~d} x \leq F_{\varepsilon}(u)+k_{0}|\Omega|+c_{1}(\kappa, p) \int_{\Omega_{\varepsilon}}\left(|\Phi|^{p}+|D \Phi|^{p}\right) \mathrm{d} x .
$$

According to $(2.5)$ we can choose $\kappa>0$ in such a way that $\left(c_{1}-k_{0}\left(1+(1+\kappa) k_{f}\right)\right)>0$. Considering (2.8) we conclude that for some $c_{1}(p)>0$ and $c_{2}(p)>0$ the inequality

$$
c_{1}(p) \int_{\Omega_{\varepsilon}}\left(|u|^{p}+|D u|^{p}\right) \mathrm{d} x \leq F_{\varepsilon}(u)+k_{0}\left|\Omega_{\varepsilon}\right|+c_{2}(p) \int_{\Omega_{\varepsilon}}\left(|\Phi|^{p}+|D \Phi|^{p}\right) \mathrm{d} x
$$

holds for all $u \in W^{1, p}\left(\Omega_{\varepsilon}\right)$ such that $u=\Phi$ on $\partial \Omega$. This completes the proof of equi-coercivity.

Another important property of $F_{\varepsilon}$ is its lower semi-continuity in the space $L^{p}(\Omega)$. To prove this property notice that, by Lemma 2.1, the surface integral $\int_{S_{\varepsilon}} g\left(\frac{x}{\varepsilon}, u\right) \mathrm{d} \mathcal{H}^{n-1}$ is uniformly in $\varepsilon$ continuous in the space $W^{1, p}$ equipped with the topology of weak convergence. This implies that the said surface integral is uniformly in $\varepsilon$ continuous with respect to the strong $L^{p}$ topology in any bounded subset of $W^{1, p}(\Omega)$. The lower semi-continuity of $F_{\varepsilon}$ is then a consequence of the assumptions of Section 1 and of the coerciveness.

Notice also that, according to Proposition 2.4, for any $u \in W^{1, p}(\Omega)$ a recovery sequence $\left\{u_{\varepsilon}\right\}$ which satisfies $\Gamma$-limsup inequality can be chosen in such a way that $\left.u_{\varepsilon}\right|_{\partial \Omega}=\left.u\right|_{\partial \Omega}$ for all $\varepsilon>0$.

The statement of Corollary 2.5 now follows from the standard properties of $\Gamma$-convergence (see, for instance [12]).

Remark 2.6. It should be noted that the functional $F_{\varepsilon}$ is coercive only in the presence of a dissipative boundary conditions on $\partial \Omega$. Indeed, in the case of the homogeneous Neumann boundary condition on $\partial \Omega$, letting $u_{\varepsilon}(x)=1 / \varepsilon$ in $\Omega$ we obtain the sequence of zero energy functions which tends to infinity as $\varepsilon \rightarrow 0$. 


\section{Preliminary Results}

We begin this section by recalling some inequalities valid in Sobolev spaces. For their proof see, for instance $[2,20]$. Under our assumptions on $E$, and $S$, there exist positive constants $k_{p}, k_{t}$ such that for each $u \in W^{1, p}(Y)$ the following inequalities hold:

Poincaré-Wirtinger inequality

$$
\int_{S \cap Y}|u-\bar{u}|^{p} \mathrm{~d} \mathcal{H}^{n-1} \leq k_{p} \int_{E \cap Y}|D u|^{p} \mathrm{~d} y
$$

where

$$
\bar{u}=|E \cap Y|^{-1} \int_{E \cap Y} u \mathrm{~d} x
$$

(the inequality remains valid if $\bar{u}$ is replaced by the surface average of $u$ on $S \cap Y$ ).

Trace inequality

$$
\int_{S \cap Y}|u|^{p} \mathrm{~d} \mathcal{H}^{n-1} \leq k_{t} \int_{E \cap Y}\left(|u|^{p}+|D u|^{p}\right) \mathrm{d} y .
$$

By performing the change of variable $y=\frac{x}{\varepsilon}$ it is easy to obtain the corresponding re-scaled estimates. Given a function $u \in W^{1, p}(\Omega)$, denote by $\bar{u}_{\varepsilon}(\cdot)$ the piecewise-constant function obtained by taking the mean value of $u$ over each cell $Y_{\varepsilon}^{i}$, i.e.,

$$
\bar{u}_{\varepsilon}(x)=\frac{1}{\left|Y_{\varepsilon}^{i} \cap \varepsilon E\right|} \int_{Y_{\varepsilon}^{i} \cap \varepsilon E} u(y) \mathrm{d} y, \quad \text { if } x \in Y_{\varepsilon}^{i} \cap \varepsilon E .
$$

Then, it is easy to check that, for every $u \in W^{1, p}(\Omega)$ and every $\varepsilon>0$

$$
\begin{gathered}
\int_{S_{\varepsilon}}\left|u-\bar{u}_{\varepsilon}\right|^{p} \mathrm{~d} \mathcal{H}^{n-1} \leq k_{p} \varepsilon^{p-1} \int_{\Omega_{\varepsilon}}|D u|^{p} \mathrm{~d} y \\
\left.\int_{S_{\varepsilon}}|u|^{p} \mathrm{~d} \mathcal{H}^{n-1} \leq k_{t}\left(\varepsilon^{-1} \int_{\Omega_{\varepsilon}}|u|^{p} \mathrm{~d} y+\varepsilon^{p-1} \int_{\Omega_{\varepsilon}}|D u|^{p}\right) \mathrm{d} y\right) .
\end{gathered}
$$

Using the preceding inequalities we can prove the statement of Lemma 2.1.

Proof of Lemma 2.1. We first prove auxiliary inequalities for $W^{1, p}(Y)$ functions. Let $u \in W^{1, p}(Y)$ and $\bar{u}$ be defined by (3.2); then, by (1.5) and (1.6) we have

$$
\begin{aligned}
\left|\int_{Y \cap S} g(y, u) \mathrm{d} \mathcal{H}^{n-1}\right| & =\left|\int_{Y \cap S}(g(y, u)-g(y, \bar{u})+g(y, \bar{u})) \mathrm{d} \mathcal{H}^{n-1}\right| \\
& \leq c_{4} \int_{S \cap Y}|u-\bar{u}|(1+|u|+|\bar{u}|)^{p-1} .
\end{aligned}
$$

By using Holder inequality, (3.7), (3.1) and (3.3) we obtain

$$
\begin{aligned}
\left|\int_{Y \cap S} g(y, u) \mathrm{d} \mathcal{H}^{n-1}\right| & \leq c_{4}\left(\int_{Y \cap S}|u-\bar{u}|^{p} \mathrm{~d} \mathcal{H}^{n-1}\right)^{\frac{1}{p}}\left(\int_{Y \cap S}(1+|u|+|\bar{u}|)^{p} \mathrm{~d} \mathcal{H}^{n-1}\right)^{\frac{1}{p^{\prime}}} \\
& \leq c_{4}\left(k_{p} \int_{Y \cap E}|D u|^{p} \mathrm{~d} y\right)^{\frac{1}{p}}\left(k_{t} \int_{Y \cap E}\left((1+|u|+|\bar{u}|)^{p}+|D u|^{p}\right) \mathrm{d} y\right)^{\frac{1}{p^{\prime}}} .
\end{aligned}
$$


Now, consider $u \in W^{1, p}\left(\Omega_{\varepsilon}\right)$. Making the change of variable $y=\frac{x}{\varepsilon}$, for each $Y_{\varepsilon}^{i} \subseteq \Omega$ we have

$$
\begin{aligned}
\varepsilon^{1-n}\left|\int_{Y_{\varepsilon}^{i} \cap S_{\varepsilon}} g\left(\frac{x}{\varepsilon}, u\right) \mathrm{d} \mathcal{H}^{n-1}\right| & \leq c\left(\varepsilon^{-n} \int_{Y_{\varepsilon}^{i} \cap \varepsilon E} \varepsilon^{p}|D u|^{p} \mathrm{~d} x\right)^{\frac{1}{p}}\left(\varepsilon^{-n} \int_{Y_{\varepsilon}^{i} \cap \varepsilon E}\left(1+|u|^{p}+\left|\bar{u}_{\varepsilon}\right|^{p}+\varepsilon^{p}|D u|^{p}\right) \mathrm{d} x\right)^{\frac{1}{p^{\prime}}} \\
& \leq c \varepsilon^{-\frac{n}{p}}\left(\int_{Y_{\varepsilon}^{i} \cap \varepsilon E} \varepsilon^{p}|D u|^{p} \mathrm{~d} x\right)^{1 / p} \cdot \varepsilon^{-\frac{n}{p^{\prime}}}\left(\int_{Y_{\varepsilon}^{i} \cap \varepsilon E}\left(\left(1+|u|+\left|\bar{u}_{\varepsilon}\right|\right)^{p}+\varepsilon^{p}|D u|^{p}\right) \mathrm{d} x\right)^{\frac{1}{p^{\prime}}} \\
& =c \varepsilon^{1-n}\left(\int_{Y_{\varepsilon}^{i} \cap \varepsilon E}|D u|^{p} \mathrm{~d} x\right)^{1 / p}\left(\int_{Y_{\varepsilon}^{i} \cap \varepsilon E}\left(\left(1+|u|+\left|\bar{u}_{\varepsilon}\right|\right)^{p}+\varepsilon^{p}|D u|^{p}\right) \mathrm{d} x\right)^{\frac{1}{p^{\prime}}} \cdot
\end{aligned}
$$

By the Young inequality, for any $\gamma>0$ we get

$$
\left|\int_{Y_{\varepsilon}^{i} \cap S_{\varepsilon}} g(y, u) \mathrm{d} \mathcal{H}^{n-1}\right| \leq \gamma \int_{Y_{\varepsilon}^{i} \cap \varepsilon E}|D u|^{p} \mathrm{~d} x+c \gamma^{-p^{\prime} / p} \int_{Y_{\varepsilon}^{i} \cap \varepsilon E}\left(\left(1+|u|+\left|\bar{u}_{\varepsilon}\right|\right)^{p}+\varepsilon^{p}|D u|^{p}\right) \mathrm{d} x .
$$

For $\bar{u}_{\varepsilon}$ Jensen's inequality yields the bound

$$
\left|\bar{u}_{\varepsilon}\right|^{p} \leq \frac{1}{\left|\varepsilon E \cap Y_{\varepsilon}^{i}\right|} \int_{\varepsilon E \cap Y_{\varepsilon}^{i}}|u|^{p} \mathrm{~d} x
$$

and we finally obtain

$$
\left|\int_{Y_{\varepsilon}^{i} \cap S_{\varepsilon}} g\left(\frac{x}{\varepsilon}, u\right) \mathrm{d} \mathcal{H}^{n-1}\right| \leq\left(\gamma+\varepsilon^{p} c(\gamma)\right) \int_{Y_{\varepsilon}^{i} \cap \varepsilon E}|D u|^{p} \mathrm{~d} x+c(\gamma) \int_{Y_{\varepsilon}^{i} \cap \varepsilon E}(1+|u|)^{p} \mathrm{~d} x .
$$

Taking the sum over $i \in I \subset I_{\varepsilon}$ or $i \in I_{\varepsilon}$ we obtain the estimates (2.1) and (2.2), respectively. The estimate (2.3) easily follows from (2.2).

We proceed to coercivity properties of functionals $F_{\varepsilon}$.

Lemma 3.1. Assume that $u_{\varepsilon} \in W^{1, p}\left(\Omega_{\varepsilon}\right)$ satisfies the bound

$$
\int_{\Omega_{\varepsilon}}\left|u_{\varepsilon}\right|^{p} \mathrm{~d} x \leq c
$$

with a constant $c>0$ independent of $\varepsilon$, and let $F_{\varepsilon}\left(u_{\varepsilon}\right) \leq c$. Then there exists $\varepsilon_{0}>0$ such that

$$
\int_{\Omega_{\varepsilon}}\left(\left|u_{\varepsilon}\right|^{p}+\left|D u_{\varepsilon}\right|^{p}\right) \mathrm{d} x \leq c^{\prime} \quad \forall \varepsilon \in\left(0, \varepsilon_{0}\right)
$$

for a suitable constant $c^{\prime}>0$ which does not depend on $\varepsilon$. In other words, there are constants $c_{1}, c_{2}>0$ and $\varepsilon_{0}>0$, such that

$$
F_{\varepsilon}(u)+c_{1}\|u\|_{L^{p}\left(\Omega_{\varepsilon}\right)}^{p} \geq c_{2}\|u\|_{W^{1, p}\left(\Omega_{\varepsilon}\right)}^{p}
$$

for all $\varepsilon<\varepsilon_{0}$.

Proof. The desired statement follows immediately from the estimate (2.2) and assumption (1.3).

Our next aim is to obtain some properties of the Lagrangian $L$, defined in (1.14). 
Proposition 3.2. The function L defined by (1.14) has the following properties:

(a) $L(z, \cdot)$ is convex for every $z \in \mathbb{R}$.

(b) $L(\cdot, \xi)$ is Lipschitz-continuous for every $\xi \in \mathbb{R}^{n}$, i.e.,

$$
\left|L\left(z_{2}, \xi\right)-L\left(z_{1}, \xi\right)\right| \leq c_{5}\left(1+\left|z_{1}\right|^{p-1}+\left|z_{2}\right|^{p-1}+|\xi|^{p-1}\right)\left|z_{1}-z_{2}\right|
$$

for every $z_{1}, z_{2} \in \mathbb{R}$ and every $\xi \in \mathbb{R}^{n}$.

(c) There are positive constants $k_{1}, k_{2}, k_{3}$ such that

$$
L(z, \xi) \geq k_{1}|\xi|^{p}-k_{2}|z|^{p}-k_{3}
$$

for every $z \in \mathbb{R}$, and every $\xi \in \mathbb{R}^{n}$.

(d) There is a positive constant $k_{4}$ such that

$$
L(z, \xi) \leq k_{4}\left(|\xi|^{p}+|z|^{p}+1\right)
$$

for every $z \in \mathbb{R}$, and every $\xi \in \mathbb{R}^{n}$.

Proof.

(a) Let us rewrite $L$ as

$$
L(z, \xi)=\int_{Y \cap S} g_{z}^{\prime}(y, z)(\xi \cdot y) \mathrm{d} \mathcal{H}^{n-1}(y)+\inf _{w}\left(\int_{Y \cap E} f(y, \xi+D w) \mathrm{d} y+\int_{Y \cap S} g_{z}^{\prime}(y, z) w \mathrm{~d} \mathcal{H}^{n-1}(y)\right) .
$$

The first term is linear in $\xi$, while the second one is easily proved to be convex, since $f(y, \xi)$ is convex with respect to $\xi$. Hence the function $L(z, \cdot)$ is convex.

(b) For brevity we denote by $\mathcal{L}$ the function

$$
\mathcal{L}(z, \xi, w)=\int_{Y \cap E} f(y, \xi+D w) \mathrm{d} y+\int_{S \cap Y} g_{z}^{\prime}(y, z)(\xi \cdot y+w) \mathrm{d} \mathcal{H}^{n-1}
$$

Let us fix $\xi \in \mathbb{R}^{n}$, and $z_{1}, z_{2} \in \mathbb{R}$ such that $L\left(z_{1}, \xi\right)<L\left(z_{2}, \xi\right)$. For every $\eta>0$ there exists $w_{\eta} \in W_{\text {per }}^{1, p}(Y \cap E)$ such that

$$
L\left(z_{1}, \xi\right)+\eta>\mathcal{L}\left(z_{1}, \xi, w_{\eta}\right)
$$

The function $w_{\eta}$ is defined up to an additive constant. Choosing this additive constant in a proper way, one can assume without loss of generality, that the mean value of either $w_{\eta}$ or $\left(\xi \cdot y+w_{\eta}\right)$ is equal to zero, so that the Poincaré inequality holds. It is not difficult to show that

$$
\left\|D w_{\eta}\right\|_{L^{p}(Y \cap E)}^{p} \leq k_{6}\left(1+|\xi|^{p}+\left|z_{1}\right|^{p}\right), \quad\left\|\xi+D w_{\eta}\right\|_{L^{p}(Y \cap E)}^{p} \leq k_{6}\left(1+|\xi|^{p}+\left|z_{1}\right|^{p}\right)
$$


with $k_{6}>0$ which does not depend on $\eta$. Indeed, by (1.3) and (3.10) we get

$$
\begin{aligned}
\int_{Y \cap E}\left|\xi+D w_{\eta}\right|^{p} \mathrm{~d} y & \leq \frac{1}{c_{1}} \int_{Y \cap E} \leq f\left(y, \xi+D w_{\eta}\right) \mathrm{d} y \\
& \leq \frac{1}{c_{1}} L\left(\xi, z_{1}\right)+\frac{1}{c_{1}}\left|\int_{S \cap Y} g_{z}^{\prime}\left(y, z_{1}\right)\left(\xi \cdot y+w_{\eta}\right) \mathrm{d} \mathcal{H}^{n-1}\right|+\frac{\eta}{c_{1}} \\
& \leq C\left(1+|\xi|^{p}+\left|z_{1}\right|^{p}\right)+C\left(1+\left|z_{1}\right|^{p-1}\right) \int_{S \cap Y}\left|\xi \cdot y+w_{\eta}\right| \mathrm{d} \mathcal{H}^{n-1} \\
& \leq C\left(1+|\xi|^{p}+\left|z_{1}\right|^{p}\right)+C\left(1+\left|z_{1}\right|^{p-1}\right)\left(\int_{Y \cap E}\left|\xi+D w_{\eta}\right|^{p} \mathrm{~d} y\right)^{1 / p} \\
& \leq C\left(1+|\xi|^{p}+\left|z_{1}\right|^{p}\right)+\frac{1}{2} \int_{Y \cap E}\left|\xi+D w_{\eta}\right|^{p} \mathrm{~d} y
\end{aligned}
$$

here we have also used the Young and the trace inequalities. This yields the second upper bound in (3.12). The first upper bound easily follows form the second one. Similar arguments are used in the proof of Proposition 3.4. The reader can find more detail proof there.

Now, by the definition of $L$, we have

$$
\begin{aligned}
0<L\left(z_{2}, \xi\right)-L\left(z_{1}, \xi\right) & \leq \mathcal{L}\left(z_{2}, \xi, w_{\eta}\right)-\mathcal{L}\left(z_{1}, \xi, w_{\eta}\right)+\eta \\
& =\int_{Y \cap S}\left(g_{z}^{\prime}\left(y, z_{2}\right)-g_{z}^{\prime}\left(y, z_{1}\right)\right)\left(\xi \cdot y+w_{\eta}\right) \mathrm{d} \mathcal{H}^{n-1}+\eta
\end{aligned}
$$

By the Lipschitz-continuity of $g_{z}^{\prime}$ (see (1.7)) we conclude that

$$
\begin{aligned}
0<L\left(z_{2}, \xi\right)-L\left(z_{1}, \xi\right) & \leq c_{5}\left(1+\left|z_{1}\right|^{p-2}+\left|z_{2}\right|^{p-2}\right)\left|z_{1}-z_{2}\right|\left\|\xi+D w_{\eta}\right\|_{L^{p}(Y \cap E)}^{1 / p}+\eta \\
& \leq c_{6}\left(1+\left|z_{1}\right|^{p-1}+\left|z_{2}\right|^{p-1}+|\xi|^{p-1}\right)\left|z_{1}-z_{2}\right|+\eta
\end{aligned}
$$

If $L\left(z_{2}, \xi\right)<L\left(z_{1}, \xi\right)$ the proof is analogous. Since $\eta$ is an arbitrary positive number, then (3.8) follows.

(c) By the definition (3.11) of $L$ and by the coercivity of $f$ (see (1.3)), for every $w \in W_{\text {per }}^{1, p}(Y \cap E$ ) with zero average on $Y \cap E$ we have the following estimate

$$
\begin{aligned}
\mathcal{L}(z, \xi, w) & \geq \int_{Y \cap E} f(y, z, \xi+D w) \mathrm{d} y+\int_{S \cap Y} g_{z}^{\prime}(y, z)(\xi \cdot y+w) \mathrm{d} \mathcal{H}^{n-1} \\
& \geq c_{1} \int_{Y \cap E}|\xi+D w|^{p} \mathrm{~d} y+\int_{S \cap Y} g_{z}^{\prime}(y, z)(\xi \cdot y+w) \mathrm{d} \mathcal{H}^{n-1} .
\end{aligned}
$$

By assumption (1.4) we can estimate the second integral above as follows

$$
\begin{aligned}
\left|\int_{S \cap Y} g_{z}^{\prime}(y, z)(\xi \cdot y+w) \mathrm{d} \mathcal{H}^{n-1}\right| & \leq c_{3} \int_{S \cap Y}\left(1+|z|^{p-1}\right)|\xi \cdot y+w| \mathrm{d} \mathcal{H}^{n-1} \\
& \leq c \mathcal{H}^{n-1}(Y \cap S)^{1-1 / p}\left(1+|z|^{p-1}\right)\left(\int_{S \cap Y}|\xi \cdot y+w|^{p} \mathrm{~d} \mathcal{H}^{n-1}\right)^{1 / p}
\end{aligned}
$$


where $c$ is a suitable positive constant, and we have applied Holder's inequality. Now, by the trace inequality (3.3)

$$
\begin{array}{r}
c \mathcal{H}^{n-1}(Y \cap S)^{1-1 / p}\left(1+|z|^{p-1}\right)\left(\int_{S \cap Y}|\xi \cdot y+w|^{p} \mathrm{~d} \mathcal{H}^{n-1}\right)^{1 / p} \leq \\
c^{\prime}\left(1+|z|^{p-1}\right)\left(\int_{E \cap Y}\left(|\xi \cdot y+w|^{p}+|\xi+D w|^{p}\right) \mathrm{d} y\right)^{1 / p} .
\end{array}
$$

By means of Poincaré inequality (3.1), we obtain also that

$c^{\prime}\left(1+|z|^{p-1}\right)\left(\int_{E \cap Y}\left(|\xi \cdot y+w|^{p}+|\xi+D w|^{p}\right) \mathrm{d} y\right)^{1 / p} \leq c^{\prime} k_{p}\left(1+|z|^{p-1}\right)\left(\int_{E \cap Y}|\xi+D w|^{p} \mathrm{~d} y\right)^{1 / p}$.

Now, by Young's inequality, for every $\eta>0$ there exists $c_{\eta}>0$ such that

$$
c^{\prime} k_{p}\left(1+|z|^{p-1}\right)\left(\int_{E \cap Y}|\xi+D w|^{p} \mathrm{~d} y\right)^{1 / p} \leq c_{\eta}\left(1+|z|^{p}\right)+\eta \int_{E \cap Y}|\xi+D w|^{p} \mathrm{~d} y .
$$

Hence, we have obtained that

$$
\left|\int_{S \cap Y} g_{z}^{\prime}(y, z)(\xi \cdot y+w) \mathrm{d} \mathcal{H}^{n-1}\right| \leq c_{\eta}\left(1+|z|^{p}\right)+\eta \int_{E \cap Y}|\xi+D w|^{p} \mathrm{~d} y .
$$

According to [1] there is an extension operator from $W^{1, p}(Y \cap E)$ to $W^{1, p}(Y)$ such that the extended function (still denoted $w$ ) satisfies the inequality

$$
\int_{Y}|\xi+D w|^{p} \mathrm{~d} y \leq C \int_{Y \cap E}|\xi+D w|^{p} \mathrm{~d} y
$$

with a constant $C$ which does not depend on $w$. Hence, using Jensen's inequality we can estimate $L$ as follows

$$
\begin{aligned}
L(z, \xi)=\inf _{w} \mathcal{L}(z, \xi, w) \geq & \inf \left(c_{1} \int_{Y \cap E}|\xi+D w|^{p} \mathrm{~d} y-\eta \int_{E \cap Y}|\xi+D w|^{p} \mathrm{~d} y\right) \\
& -c\left(1+|z|^{p}\right) \geq \inf \left(\frac{c_{1}}{C} \int_{Y}|\xi+D w|^{p} \mathrm{~d} y-\eta \int_{Y}|\xi+D w|^{p} \mathrm{~d} y\right) \\
& -c\left(1+|z|^{p}\right) \geq\left(\frac{c_{1}}{C}-\eta\right) \int_{Y}|\xi|^{p} \mathrm{~d} y-c\left(1+|z|^{p}\right) .
\end{aligned}
$$

From the arbitrariness of $\eta$ inequality (3.9) follows immediately.

(d) This upper bound is straightforward. Indeed, it suffices to substitute $w=0$ in the definition of $L(\xi, z)$, and the required bound follows from (1.3)-(1.4).

Remark 3.3. In the case of disjoint inclusions studied here, one can improve the upper bound (3.10) for $L(\xi, z)$ by choosing the test function $w(y)$ equal to $-\xi \cdot y$ on $S$ and zero on $\partial Y$, in such a way that $|\nabla w| \leq C|\xi|$. This yields an upper bound

$$
L(\xi, z) \leq k_{5}\left(|\xi|^{p}+1\right)
$$

with a positive constant $k_{5}$. However, having in mind more general case of connected perforation, we prefer not to use this estimate. 
Proposition 3.4. For every $z \in \mathbb{R}, \xi \in \mathbb{R}^{n}$, there exists at least one solution $w(\cdot, z, \xi) \in W^{1, p}(Y \cap E)$ to the minimum problem (1.14). Moreover, for every $k \in \mathbb{R}$ the function $w+k$ is also a solution. Finally, there exists a positive constant $c_{0}$ such that

$$
\begin{gathered}
\int_{Y \cap E}|w(\cdot, z, \xi)|^{p} \mathrm{~d} y \leq c_{0}\left(1+|z|^{p}+|\xi|^{p}\right), \\
\int_{Y \cap E}|D w(\cdot, z, \xi)|^{p} \mathrm{~d} y \leq c_{0}\left(1+|z|^{p}+|\xi|^{p}\right)
\end{gathered}
$$

for every $z \in \mathbb{R}, \xi \in \mathbb{R}^{n}$, and for every solution $w$ that has zero average on the set $Y \cap E$.

Proof. Let $\mathcal{L}(z, \xi, w)$ be defined by (3.11) for every $z \in \mathbb{R}, \xi \in \mathbb{R}^{n}, w \in W^{1, p}(Y \cap E)$. It is easy to see that $\mathcal{L}(z, \xi, \cdot)$ is lower-semicontinuous on $W^{1, p}(Y \cap E)$ with respect to $W^{1, p}$-weak convergence. Moreover, $\mathcal{L}(z, \xi, \cdot)$ is also coercive; in fact, using (1.3) and (3.13), one can show that, for every $\eta>0$ there exist $c_{\eta}$ such that

$$
\mathcal{L}(z, \xi, w) \geq\left(c_{1}-\eta\right) \int_{Y \cap E}|\xi+D w|^{p} \mathrm{~d} y-2 c_{\eta}\left(1+|z|^{p}\right)
$$

for any $w$ with zero average. Therefore, problem (1.14) has at least one solution. If $w$ is a solution and $k \in \mathbb{R}$, then also $w+k$ is a solution to problem (1.14) since, by (1.5)

$$
\int_{Y \cap S} g_{z}^{\prime}(y, z) \mathrm{d} \mathcal{H}^{n-1}=0 \text { for all } z \in \mathbb{R}
$$

In order to prove estimate (3.14), let $w \in W^{1, p}(Y \cap E)$ be a solution of (1.14) with zero mean value

$$
\int_{Y \cap E} w \mathrm{~d} y=0
$$

By definition, $\mathcal{L}(z, \xi, w) \leq \mathcal{L}(z, \xi, 0)$ and hence

$$
\int_{Y \cap E} f(y, z, \xi+D w) \mathrm{d} y+\int_{Y \cap S} g_{z}^{\prime}(y, z) w \mathrm{~d} \mathcal{H}^{n-1} \leq \int_{Y \cap E} f(y, z, \xi) \mathrm{d} y .
$$

By (3.16) we obtain that for every $\eta>0$ there exists $c_{\eta}>0$, such that

$$
\left(c_{1}-\eta\right) \int_{Y \cap E}|\xi+D w|^{p} \mathrm{~d} y-c_{\eta}\left(1+|z|^{p}\right) \leq c_{2}\left(1+|z|^{p}+|\xi|^{p}\right)|Y \cap E| .
$$

From this inequality (3.15) follows easily. The bound (3.14) follows, thanks to the Poincaré inequality.

We now state a lemma, that will be used both in the proof of $\Gamma$-lim inf and $\Gamma$-lim sup inequality.

Lemma 3.5. There exists a constant $c>0$ such that if $u_{\varepsilon} \in W^{1, p}\left(\Omega_{\varepsilon}\right)$ and $\bar{u}_{\varepsilon}$ is the piece-wise constant function defined by (3.4) as the integral average of $u_{\varepsilon}$ in each cell $Y_{\varepsilon}^{i}$, then

$$
\left|\int_{S_{\varepsilon}} g\left(\frac{x}{\varepsilon}, u_{\varepsilon}\right) \mathrm{d} \mathcal{H}^{n-1}-\int_{S_{\varepsilon}} g_{z}^{\prime}\left(\frac{x}{\varepsilon}, \bar{u}_{\varepsilon}\right)\left(u_{\varepsilon}-\bar{u}_{\varepsilon}\right) \mathrm{d} \mathcal{H}^{n-1}\right| \leq c \max \left\{\varepsilon, \varepsilon^{p-1}\right\}\left(1+\int_{\Omega_{\varepsilon}}\left(\left|u_{\varepsilon}\right|^{p}+\left|D u_{\varepsilon}\right|^{p}\right) \mathrm{d} x\right)
$$

for all $\varepsilon>0$. 
Proof. We make use of the representation

$$
\int_{S_{\varepsilon}} g\left(\frac{x}{\varepsilon}, u_{\varepsilon}\right) \mathrm{d} \mathcal{H}^{n-1}=\sum_{i \in I_{\varepsilon}} \int_{S_{\varepsilon} \cap Y_{\varepsilon}^{i}} g\left(\frac{x}{\varepsilon}, u_{\varepsilon}\right) \mathrm{d} \mathcal{H}^{n-1}
$$

and estimate separately the integral over different cells $S_{\varepsilon} \cap Y_{\varepsilon}^{i}$. Since $\bar{u}_{\varepsilon}$ is constant in $Y_{\varepsilon}^{i}$, by $(1.5)$ and the regularity of $g(y, \cdot)$ we have

$$
\begin{aligned}
\int_{S_{\varepsilon} \cap Y_{\varepsilon}^{i}} g\left(\frac{x}{\varepsilon}, u_{\varepsilon}\right) \mathrm{d} \mathcal{H}^{n-1} & =\int_{S_{\varepsilon} \cap Y_{\varepsilon}^{i}} g\left(\frac{x}{\varepsilon}, \bar{u}_{\varepsilon}+\left(u_{\varepsilon}-\bar{u}_{\varepsilon}\right)\right) \mathrm{d} \mathcal{H}^{n-1} \\
& =\int_{0}^{1} \int_{S_{\varepsilon} \cap Y_{\varepsilon}^{i}} g_{z}^{\prime}\left(\frac{x}{\varepsilon}, \bar{u}_{\varepsilon}+t\left(u_{\varepsilon}-\bar{u}_{\varepsilon}\right)\right)\left(u_{\varepsilon}-\bar{u}_{\varepsilon}\right) \mathrm{d} \mathcal{H}^{n-1} \mathrm{~d} t .
\end{aligned}
$$

From (1.7) we have

$$
\begin{aligned}
\left|\int_{0}^{1} \int_{S_{\varepsilon} \cap Y_{\varepsilon}^{i}}\left[g_{z}^{\prime}\left(\frac{x}{\varepsilon}, \bar{u}_{\varepsilon}+t\left(u_{\varepsilon}-\bar{u}_{\varepsilon}\right)\right)-g_{z}^{\prime}\left(\frac{x}{\varepsilon}, \bar{u}_{\varepsilon}\right)\right]\left(u_{\varepsilon}-\bar{u}_{\varepsilon}\right) \mathrm{d} \mathcal{H}^{n-1} \mathrm{~d} t\right| \leq \\
c_{5} \int_{S_{\varepsilon} \cap Y_{\varepsilon}^{i}}\left(1+\left|u_{\varepsilon}\right|+\left|\bar{u}_{\varepsilon}\right|\right)^{p-2}\left|u_{\varepsilon}-\bar{u}_{\varepsilon}\right|^{2} \mathrm{~d} \mathcal{H}^{n-1} .
\end{aligned}
$$

If $p \geq 2$ then by applying Holder's inequality we get

$$
\begin{gathered}
\int_{S_{\varepsilon} \cap Y_{\varepsilon}^{i}}\left(1+\left|u_{\varepsilon}\right|+\left|\bar{u}_{\varepsilon}\right|\right)^{p-2}\left|u_{\varepsilon}-\bar{u}_{\varepsilon}\right|^{2} \mathrm{~d} \mathcal{H}^{n-1} \leq \\
c\left(\int_{S_{\varepsilon} \cap Y_{\varepsilon}^{i}}\left|u_{\varepsilon}-\bar{u}_{\varepsilon}\right|^{p} \mathrm{~d} \mathcal{H}^{n-1}\right)^{2 / p} \cdot\left(\int_{S_{\varepsilon} \cap Y_{\varepsilon}^{i}}\left(1+\left|u_{\varepsilon}\right|^{p}+\left|\bar{u}_{\varepsilon}\right|^{p}\right) \mathrm{d} \mathcal{H}^{n-1}\right)
\end{gathered}
$$

Since we can estimate

$$
\int_{S_{\varepsilon} \cap Y_{\varepsilon}^{i}}\left|\bar{u}_{\varepsilon}\right|^{p} \mathrm{~d} \mathcal{H}^{n-1} \leq c \varepsilon^{-1} \int_{\Omega_{\varepsilon} \cap Y_{\varepsilon}^{i}}\left|\bar{u}_{\varepsilon}\right|^{p} \mathrm{~d} x \leq c \varepsilon^{-1} \int_{\Omega_{\varepsilon} \cap Y_{\varepsilon}^{i}}\left|u_{\varepsilon}\right|^{p} \mathrm{~d} x,
$$

then by (3.5), (3.6) we have

$$
\begin{aligned}
&\left(\int_{S_{\varepsilon} \cap Y_{\varepsilon}^{i}}\left|u_{\varepsilon}-\bar{u}_{\varepsilon}\right|^{p} \mathrm{~d} \mathcal{H}^{n-1}\right)^{2 / p} \cdot\left(\int_{S_{\varepsilon} \cap Y_{\varepsilon}^{i}}\left(1+\left|u_{\varepsilon}\right|^{p}+\left|\bar{u}_{\varepsilon}\right|^{p}\right) \mathrm{d} \mathcal{H}^{n-1}\right)^{\frac{p-2}{p}} \leq \\
& c\left(\varepsilon^{p-1} \int_{\Omega_{\varepsilon} \cap Y_{\varepsilon}^{i}}\left|D u_{\varepsilon}\right|^{p} \mathrm{~d} x\right)^{2 / p} \cdot\left(\varepsilon^{-1} \int_{\Omega_{\varepsilon} \cap Y_{\varepsilon}^{i}}\left(1+\left|u_{\varepsilon}\right|^{p}\right) \mathrm{d} x+\varepsilon^{p-1} \int_{\Omega_{\varepsilon} \cap Y_{\varepsilon}^{i}}\left|D u_{\varepsilon}\right|^{p} \mathrm{~d} x\right)^{\frac{p-2}{p}} \\
&=c \varepsilon^{p-1} \int_{\Omega_{\varepsilon} \cap Y_{\varepsilon}^{i}}\left|D u_{\varepsilon}\right|^{p} \mathrm{~d} x+c \varepsilon\left(\int_{\Omega_{\varepsilon} \cap Y_{\varepsilon}^{i}}\left|D u_{\varepsilon}\right|^{p} \mathrm{~d} x\right)^{2 / p} \cdot\left(\int_{\Omega_{\varepsilon} \cap Y_{\varepsilon}^{i}}\left(1+\left|u_{\varepsilon}\right|^{p}\right) \mathrm{d} x\right)^{\frac{p-2}{p}} .
\end{aligned}
$$


By Young's inequality with powers $p / 2$ and $(p-2) / p$ for any $t \in[0,1]$ we finally get

$$
\begin{aligned}
\left|\int_{S_{\varepsilon} \cap Y_{\varepsilon}^{i}}\left[g_{z}^{\prime}\left(\frac{x}{\varepsilon}, \bar{u}_{\varepsilon}+t\left(u_{\varepsilon}-\bar{u}_{\varepsilon}\right)\right)-g_{z}^{\prime}\left(\frac{x}{\varepsilon}, \bar{u}_{\varepsilon}\right)\right]\left(u_{\varepsilon}-\bar{u}_{\varepsilon}\right) \mathrm{d} \mathcal{H}^{n-1}\right| \leq \\
\quad c \max \left\{\varepsilon^{p-1}, \varepsilon\right\} \int_{\Omega_{\varepsilon} \cap Y_{\varepsilon}^{i}}\left|D u_{\varepsilon}\right|^{p} \mathrm{~d} x+c \varepsilon \int_{\Omega_{\varepsilon} \cap Y_{\varepsilon}^{i}}\left(1+\left|u_{\varepsilon}\right|^{p}\right) \mathrm{d} x .
\end{aligned}
$$

By summing up over $i$ we complete the proof in the case $p \geq 2$.

If $1<p<2$, then

$$
\begin{aligned}
& \int_{S_{\varepsilon} \cap Y_{\varepsilon}^{i}}\left(1+\left|u_{\varepsilon}\right|+\left|\bar{u}_{\varepsilon}\right|\right)^{p-2}\left|u_{\varepsilon}-\bar{u}_{\varepsilon}\right|^{2} \mathrm{~d} \mathcal{H}^{n-1}= \\
& \int_{S_{\varepsilon} \cap Y_{\varepsilon}^{i}} \frac{\left|u_{\varepsilon}-\bar{u}_{\varepsilon}\right|^{2-p}}{\left(1+\left|u_{\varepsilon}\right|+\left|\bar{u}_{\varepsilon}\right|\right)^{2-p}}\left|u_{\varepsilon}-\bar{u}_{\varepsilon}\right|^{p} \mathrm{~d} \mathcal{H}^{n-1} \leq c \int_{S_{\varepsilon} \cap Y_{\varepsilon}^{i}}\left|u_{\varepsilon}-\bar{u}_{\varepsilon}\right|^{p} \mathrm{~d} \mathcal{H}^{n-1} \\
& \leq c \varepsilon^{p-1} \int_{\Omega_{\varepsilon} \cap Y_{\varepsilon}^{i}}\left|D u_{\varepsilon}\right|^{p} \mathrm{~d} x .
\end{aligned}
$$

Summing up over $i$ we obtain the desired bound.

Lemma 3.6. The family of functionals $F_{\varepsilon}$ is continuous with respect to the strong topology of $W^{1, p}(\Omega)$, uniformly with respect to $\varepsilon$.

Proof. We can prove separately that volume integral

$$
F_{\varepsilon}^{v}(u)=\int_{\Omega_{\varepsilon}} f\left(\frac{x}{\varepsilon}, u, D u\right) \mathrm{d} x
$$

and surface integral

$$
F_{\varepsilon}^{s}(u)=\int_{S_{\varepsilon}} g\left(\frac{x}{\varepsilon}, u\right) \mathrm{d} \mathcal{H}^{n-1}
$$

are continuous, uniformly with respect to $\varepsilon$. We first proceed with $F_{\varepsilon}^{v}$. From the growth and convexity conditions stated in Section 1 it follows that there exists a constant $c>0$ such that

$$
|f(y, \xi)-f(y, \eta)| \leq c\left(1+|\xi|^{p-1}+|\eta|^{p-1}\right)|\xi-\eta|
$$

for a.e. $y \in \mathbb{R}^{n}$, and every $\xi, \eta \in \mathbb{R}^{n}$. Therefore,

$$
\begin{aligned}
\left|F_{\varepsilon}^{v}(u)-F_{\varepsilon}^{v}(u)\right| & \leq c \int_{\Omega_{\varepsilon}}\left(1+|D u|^{p-1}+|D w|^{p-1}\right)|D u-D w| \mathrm{d} x \\
& \leq c\left(\int_{\Omega_{\varepsilon}}\left(1+|D u|^{p}+|D w|^{p}\right) \mathrm{d} x\right)^{\frac{p-1}{p}} \cdot\left(\int_{\Omega_{\varepsilon}}|D u-D w|^{p} \mathrm{~d} x\right)^{\frac{1}{p}} \\
& \leq c\left(1+\left\|\left.u\right|_{W^{1, p}\left(\Omega_{\varepsilon}\right)} ^{p-1}+|| w\right\|_{W^{1, p}\left(\Omega_{\varepsilon}\right)}^{p-1}\right)|| u-w \|_{W^{1, p}\left(\Omega_{\varepsilon}\right)} .
\end{aligned}
$$

Now we consider the surface integral $F_{\varepsilon}^{s}$. By Lemma 3.5 , we have

$$
\begin{aligned}
\left|F_{\varepsilon}^{s}(u)-F_{\varepsilon}^{s}(w)\right| \leq & \left|\int_{S_{\varepsilon}} g_{z}^{\prime}\left(\frac{x}{\varepsilon}, \bar{u}\right)(u-\bar{u}-(w-\bar{w})) \mathrm{d} \mathcal{H}^{n-1}\right| \\
& +\left|\int_{S_{\varepsilon}}\left(g_{z}^{\prime}\left(\frac{x}{\varepsilon}, \bar{u}\right)-g_{z}^{\prime}\left(\frac{x}{\varepsilon}, \bar{w}\right)\right)(w-\bar{w}) \mathrm{d} \mathcal{H}^{n-1}\right| \\
& +c \max \left\{\varepsilon, \varepsilon^{p-1}\right\}\left(1+\|u\|_{W^{1, p}\left(\Omega_{\varepsilon}\right)}^{p}+\|w\|_{W^{1, p}\left(\Omega_{\varepsilon}\right)}^{p}\right) .
\end{aligned}
$$


For the first integral on the right hand side, by Poincaré inequality we obtain

$$
\begin{aligned}
& \left|\int_{S_{\varepsilon}} g_{z}^{\prime}\left(\frac{x}{\varepsilon}, \bar{u}\right)(u-\bar{u}-(w-\bar{w})) \mathrm{d} \mathcal{H}^{n-1}\right| c\left(\int_{S_{\varepsilon}}\left(1+|u|^{p-1}\right) \mathrm{d} \mathcal{H}^{n-1}\right)^{\frac{p-1}{p}}\left(\int_{S_{\varepsilon}}|u-\bar{u}-(w-\bar{w})|^{p} \mathrm{~d} \mathcal{H}^{n-1}\right)^{\frac{1}{p}} \leq \\
& c\left(1+\|u\|_{W^{1, p}\left(\Omega_{\varepsilon}\right)}^{p-1}\right)\|u-w\|_{W^{1, p}\left(\Omega_{\varepsilon}\right)} .
\end{aligned}
$$

The second term on the right hand side of (3.19) can be estimated in a similar way, in view of the Lipschitz continuity of $g_{z}^{\prime}(y, \cdot)$. Combining the above bounds and taking into account the fact that for $\varepsilon \in\left(\varepsilon_{0}, 1\right), \varepsilon_{0}>0$, the uniform continuity trivially follows from the trace inequality, we obtain the desired uniform continuity for all $\varepsilon \in(0,1)$.

\section{Proof of Theorem 2.3: the $\Gamma$-Lim inf inequality}

By Definition 2.2, the family $F_{\varepsilon}(\cdot) \Gamma$-converges to a functional $F=F(u)$ if the following two properties hold:

(1) ( $\Gamma$-lim inf inequality). For any sequence $\left\{u_{\varepsilon}\right\}, u_{\varepsilon} \in L^{p}(\Omega)$, such that $u_{\varepsilon}$ converges to $u$ in $L^{p}(\Omega)$, we have

$$
\liminf _{\varepsilon \rightarrow 0} F_{\varepsilon}\left(u_{\varepsilon}\right) \geq F(u)
$$

(by $(1.2)$ ), if $u_{\varepsilon} \notin W^{1, p}\left(\Omega_{\varepsilon}\right)$, we set $\left.F_{\varepsilon}\left(u_{\varepsilon}\right)=+\infty\right)$.

(2) ( $\Gamma$-lim sup inequality). For any $u \in L^{p}(\Omega)$ there is a sequence $u_{\varepsilon} \in L^{p}(\Omega)$ such that $u_{\varepsilon}$ converges to $u$ in $L^{p}(\Omega)$ and

$$
\limsup _{\varepsilon \rightarrow 0} F_{\varepsilon}\left(u_{\varepsilon}\right) \leq F(u) \text {. }
$$

In this section we are going to prove the $\Gamma$-lim inf inequality. To this end, given any $u, u_{\varepsilon} \in W^{1, p}(\Omega)$ such that $u_{\varepsilon} \rightarrow u$ strongly in $L^{p}(\Omega)$, we have to show that

$$
F(u) \leq \liminf _{\varepsilon \rightarrow 0} F_{\varepsilon}\left(u_{\varepsilon}\right)
$$

Since $F$ is continuous on $W^{1, p}(\Omega)$ and $F_{\varepsilon}$ is continuous on $W^{1, p}(\Omega)$ uniformly with respect to $\varepsilon$ (see Lem. 3.6), then it is sufficient to prove the above inequality for piecewise affine function $u$. Moreover, without loss of generality we can assume that $F_{\varepsilon}\left(u_{\varepsilon}\right)$ has a finite limit, as $\varepsilon \rightarrow 0$. Then, by Lemma 3.1,

$$
\sup \left\{\left\|u_{\varepsilon}\right\|_{W^{1, p}\left(\Omega_{\varepsilon}\right)}: \varepsilon>0\right\}<+\infty .
$$

As we said in Section 1, by the extension theorem in [1] we can assume that $u_{\varepsilon}$ are also bounded in $W^{1, p}(\Omega)$. For any open subset $A \subset \Omega$ we denote

$$
F_{\varepsilon}(u, A)=\int_{\Omega_{\varepsilon} \cap A} f\left(\frac{x}{\varepsilon}, D u\right) \mathrm{d} x+\int_{S_{\varepsilon} \cap A} g\left(\frac{x}{\varepsilon}, u\right) \mathrm{d} \mathcal{H}^{n-1} .
$$

Step 1. Let $\bar{u}_{\varepsilon}$ be defined by (3.4) as the integral average of $u_{\varepsilon}$ in each cell $Y_{\varepsilon}^{i}$. Then, by Lemma 3.5,

$$
\begin{aligned}
\left|\int_{S_{\varepsilon}} g\left(\frac{x}{\varepsilon}, u_{\varepsilon}\right) \mathrm{d} \mathcal{H}^{n-1}-\int_{S_{\varepsilon}} g_{z}^{\prime}\left(\frac{x}{\varepsilon}, \bar{u}_{\varepsilon}\right)\left(u_{\varepsilon}-\bar{u}_{\varepsilon}\right) \mathrm{d} \mathcal{H}^{n-1}\right| \leq \\
c \max \left\{\varepsilon, \varepsilon^{p-1}\right\}\left(1+\int_{\Omega_{\varepsilon}}\left(\left|u_{\varepsilon}\right|^{p}+\left|D u_{\varepsilon}\right|^{p}\right) \mathrm{d} x\right) \leq c^{\prime} \max \left\{\varepsilon, \varepsilon^{p-1}\right\}
\end{aligned}
$$

for all $\varepsilon>0$. 
Step 2. Let $A^{\delta}$ be the periodic grid with period $\delta$ and thickness $\tilde{\delta}, \tilde{\delta}=\delta^{2}+o\left(\delta^{2}\right)$, defined by $A^{\delta}=\mathbb{R}^{n} \backslash \cup\{\delta \alpha+$ $\left.\left[\frac{-\delta+\tilde{\delta}}{2}, \frac{\delta-\tilde{\delta}}{2}\right]^{n}: \alpha \in \mathbb{Z}^{n}\right\}$. We assume in what follows that both $\delta$ and $\tilde{\delta}$ are integer multipliers of $\varepsilon$. Covering the domain $\Omega$ by $\tilde{\delta}$ shifts of $A^{\delta}$ in each coordinate directions so that

$$
\Omega=\bigcup_{\alpha \in \mathbb{Z}^{n} \cap[0, \delta / \tilde{\delta}]^{n}}\left(\left(A^{\delta}+\alpha \tilde{\delta}\right) \cap \Omega\right),
$$

one can conclude in the standard way that for every $\varepsilon>0$ there exists $x_{\varepsilon}^{\delta} \in \mathbb{R}^{n}$ such that

$$
\begin{gathered}
F_{\varepsilon}\left(u_{\varepsilon},\left(A^{\delta}+x_{\varepsilon}^{\delta}\right) \cap \Omega\right) \leq c_{n} \delta F_{\varepsilon}\left(u_{\varepsilon}, \Omega\right), \\
\int_{\left(A^{\delta}+x_{\varepsilon}^{\delta}\right) \cap \Omega}\left(\left|D u_{\varepsilon}\right|^{p}+\left|u_{\varepsilon}\right|^{p}\right) \mathrm{d} x \leq c_{n} \delta \int_{\Omega}\left(\left|D u_{\varepsilon}\right|^{p}+\left|u_{\varepsilon}\right|^{p}\right) \mathrm{d} x
\end{gathered}
$$

where the constant $c_{n}>0$ only depend on the dimension $n$. Moreover, the shift $x_{\varepsilon}^{\delta}$ can be chosen in such a way that $\left(A^{\delta}+x_{\varepsilon}^{\delta}\right)$ is compatible with $\varepsilon$-grid, i.e. it consists of integer number of solid periods $\left\{Y_{\varepsilon}^{i}\right\}$. From now on, we set $A_{\varepsilon}^{\delta}=A^{\delta}+x_{\varepsilon}^{\delta}$. In view of Lemmas 2.1 and 3.1 the estimate (4.3) yields

$$
\int_{\left(A^{\delta}+x_{\varepsilon}^{\delta}\right) \cap \Omega}\left(\left|u_{\varepsilon}\right|^{p}+\left|D u_{\varepsilon}\right|^{p}\right) \mathrm{d} x \leq c_{n} \delta\left(F_{\varepsilon}\left(u_{\varepsilon}, \Omega\right)+C\right)
$$

for every $\delta>0$.

Step 3. Let $\varphi_{\varepsilon}^{\delta}$ be a $\delta$-periodic cut-off function associated to the set $A_{\varepsilon}^{\delta}$, i.e., $\varphi_{\varepsilon}^{\delta} \in \mathcal{C}_{\text {per }}^{\infty}\left(\mathbb{R}^{n}\right), 0 \leq \varphi_{\varepsilon}^{\delta} \leq 1, \varphi_{\varepsilon}^{\delta}=1$ in $\mathbb{R}^{n} \backslash A_{\varepsilon}^{\delta}, \varphi_{\varepsilon}^{\delta}=0$ on $\cup\left(\left\{\delta \alpha+\partial\left(\left[-\frac{\delta}{2}, \frac{\delta}{2}\right]^{n}\right): \alpha \in \mathbb{Z}^{n}\right\}+x_{\varepsilon}^{\delta}\right)$, and $\left|D \varphi_{\varepsilon}^{\delta}\right| \leq c / \delta^{2}$. Let us define

$$
u_{\varepsilon}^{\delta}=u+\varphi_{\varepsilon}^{\delta}\left(u_{\varepsilon}-u\right) .
$$

Then $u_{\varepsilon}^{\delta}=u$ on the boundary of each $\delta$-cell $\delta \alpha+\left[-\frac{\delta}{2}, \frac{\delta}{2}\right]^{n}+x_{\varepsilon}^{\delta}$. For the volume integral we claim that

$$
\begin{array}{r}
\left|\int_{\Omega_{\varepsilon}} f\left(\frac{x}{\varepsilon}, D u_{\varepsilon}\right) \mathrm{d} x-\int_{\Omega_{\varepsilon}} f\left(\frac{x}{\varepsilon}, D u_{\varepsilon}^{\delta}\right) \mathrm{d} x\right|=\int_{A_{\varepsilon}^{\delta}}\left|f\left(\frac{x}{\varepsilon}, D u_{\varepsilon}\right)-f\left(\frac{x}{\varepsilon}, D u_{\varepsilon}^{\delta}\right)\right| \mathrm{d} x \\
\leq c \int_{A_{\varepsilon}^{\delta}}\left(1+|D u|^{p}+\left|D u_{\varepsilon}\right|^{p}+\frac{1}{\delta^{2}}\left|u_{\varepsilon}-u\right|^{p}\right) \mathrm{d} x \\
\leq c \delta \int_{\Omega_{\varepsilon}}\left(|D u|^{p}+\left|D u_{\varepsilon}\right|^{p}+1\right) \mathrm{d} x+\frac{1}{\delta^{2}} \int_{\Omega_{\varepsilon}}\left|u_{\varepsilon}-u\right|^{p} \mathrm{~d} x \leq c \delta+\frac{1}{\delta^{2}} \kappa_{0}(\varepsilon)
\end{array}
$$

with $\kappa_{0}(\varepsilon) \rightarrow 0$ as $\varepsilon \rightarrow 0$, uniformly in $\delta$.

Step 4. Let $x_{\delta}^{\alpha}$ be the center of the cell $Y_{\delta}^{\alpha}=\delta \alpha+x_{\varepsilon}^{\delta}+\left[-\frac{\delta}{2}, \frac{\delta}{2}\right]$, and let

$$
u_{\delta}(x)=\sum_{i} u\left(x_{\delta}^{i}\right) \chi_{Y_{\delta}^{i}}(x), \quad \forall x \in \mathbb{R}^{n} .
$$

We claim that

$$
\left|\int_{S_{\varepsilon}} g_{z}^{\prime}\left(\frac{x}{\varepsilon}, \bar{u}_{\varepsilon}\right)\left(u_{\varepsilon}-\bar{u}_{\varepsilon}\right) \mathrm{d} \mathcal{H}^{n-1}-\int_{S_{\varepsilon}} g_{z}^{\prime}\left(\frac{x}{\varepsilon}, u_{\delta}\right) u_{\varepsilon}^{\delta} \mathrm{d} \mathcal{H}^{n-1}\right| \leq c\left(\delta+\delta^{1 / p}+\kappa(\varepsilon)\left(1+\frac{1}{\delta^{2}}\right)\right)
$$

with $\kappa(\varepsilon) \rightarrow 0$ as $\varepsilon \rightarrow 0$. 
First we estimate

$$
\begin{gathered}
\left|\int_{S_{\varepsilon}} g_{z}^{\prime}\left(\frac{x}{\varepsilon}, \bar{u}_{\varepsilon}\right)\left(u_{\varepsilon}-\bar{u}_{\varepsilon}\right) \mathrm{d} \mathcal{H}^{n-1}-\int_{S_{\varepsilon}} g_{z}^{\prime}\left(\frac{x}{\varepsilon}, u_{\delta}\right)\left(u_{\varepsilon}-\bar{u}_{\varepsilon}\right) \mathrm{d} \mathcal{H}^{n-1}\right| \leq \\
\int_{S_{\varepsilon}}\left(1+\left|\bar{u}_{\varepsilon}\right|+\left|u_{\delta}\right|\right)^{p-2}\left|\bar{u}_{\varepsilon}-u_{\delta}\right|\left|u_{\varepsilon}-\bar{u}_{\varepsilon}\right| \mathrm{d} \mathcal{H}^{n-1} .
\end{gathered}
$$

If $p \geq 2$ then by the Holder inequality, (3.3), and the fact that $\bar{u}_{\varepsilon}$ is constant in each $\varepsilon$-cell, we obtain

$$
\begin{aligned}
\int_{S_{\varepsilon}}\left(1+\left|\bar{u}_{\varepsilon}\right|\right. & \left.+\left|u_{\delta}\right|\right)^{p-2}\left|\bar{u}_{\varepsilon}-u_{\delta}\right|\left|u_{\varepsilon}-\bar{u}_{\varepsilon}\right| \mathrm{d} \mathcal{H}^{n-1} \leq \\
& \left(\int_{S_{\varepsilon}}\left(1+\left|\bar{u}_{\varepsilon}\right|+\left|u_{\delta}\right|\right)^{p} \mathrm{~d} \mathcal{H}^{n-1}\right)^{\frac{p-2}{p}}\left(\int_{S_{\varepsilon}}\left|\bar{u}_{\varepsilon}-u_{\delta}\right|^{p} \mathrm{~d} \mathcal{H}^{n-1}\right)^{\frac{1}{p}}\left(\int_{S_{\varepsilon}}\left|u_{\varepsilon}-\bar{u}_{\varepsilon}\right|^{p} \mathrm{~d} \mathcal{H}^{n-1}\right)^{\frac{1}{p}} \\
& \leq\left(c \varepsilon^{-1} \int_{\Omega_{\varepsilon}}\left(1+\left|\bar{u}_{\varepsilon}\right|+\left|u_{\delta}\right|\right)^{p} \mathrm{~d} x\right)^{\frac{p-2}{p}}\left(\varepsilon^{-1} \int_{\Omega_{\varepsilon}}\left|\bar{u}_{\varepsilon}-u_{\delta}\right|^{p} \mathrm{~d} x\right)^{\frac{1}{p}}\left(\varepsilon^{p-1} \int_{\Omega_{\varepsilon}}\left|D u_{\varepsilon}\right|^{p} \mathrm{~d} x\right)^{\frac{1}{p}} \\
& \leq c\left(\int_{\Omega_{\varepsilon}}\left|\bar{u}_{\varepsilon}-u_{\delta}\right|^{p} \mathrm{~d} x\right)^{\frac{1}{p}} \leq c\left(\int_{\Omega_{\varepsilon}}\left|\bar{u}_{\varepsilon}-u\right|^{p} \mathrm{~d} x\right)^{\frac{1}{p}}+c\left(\int_{\Omega_{\varepsilon}}\left|u-u_{\delta}\right|^{p} \mathrm{~d} x\right)^{\frac{1}{p}} \leq \kappa_{1}(\varepsilon)+c_{1} \delta
\end{aligned}
$$

where $\kappa_{1}(\varepsilon) \rightarrow 0$ as $\varepsilon \rightarrow 0$.

If $1<p<2$, for the same reason we have

$$
\begin{aligned}
\int_{S_{\varepsilon}}\left(1+\left|\bar{u}_{\varepsilon}\right|\right. & \left.+\left|u_{\delta}\right|\right)^{p-2}\left|\bar{u}_{\varepsilon}-u_{\delta}\right|\left|u_{\varepsilon}-\bar{u}_{\varepsilon}\right| \mathrm{d} \mathcal{H}^{n-1} \leq \\
& \int_{S_{\varepsilon}} \frac{\left|\bar{u}_{\varepsilon}-u_{\delta}\right|^{2-p}}{\left(1+\left|\bar{u}_{\varepsilon}\right|+\left|u_{\delta}\right|\right)^{2-p}}\left|\bar{u}_{\varepsilon}-u_{\delta}\right|^{p-1}\left|u_{\varepsilon}-\bar{u}_{\varepsilon}\right| \mathrm{d} \mathcal{H}^{n-1} \leq \int_{S_{\varepsilon}}\left|\bar{u}_{\varepsilon}-u_{\delta}\right|^{p-1}\left|u_{\varepsilon}-\bar{u}_{\varepsilon}\right| \mathrm{d} \mathcal{H}^{n-1} \\
& \leq\left(\int_{S_{\varepsilon}}\left|\bar{u}_{\varepsilon}-u_{\delta}\right|^{p} \mathrm{~d} \mathcal{H}^{n-1}\right)^{\frac{p-1}{p}}\left(\int_{S_{\varepsilon}}\left|u_{\varepsilon}-\bar{u}_{\varepsilon}\right|^{p} \mathrm{~d} \mathcal{H}^{n-1}\right)^{\frac{1}{p}} \\
& \leq\left(\varepsilon^{-1} \int_{\Omega_{\varepsilon}}\left|\bar{u}_{\varepsilon}-u_{\delta}\right|^{p} \mathrm{~d} x\right)^{\frac{p-1}{p}}\left(\varepsilon^{p-1} \int_{\Omega_{\varepsilon}}\left|D u_{\varepsilon}\right|^{p} \mathrm{~d} x\right)^{\frac{1}{p}} \leq \kappa_{2}(\varepsilon)+c \delta^{1-\frac{1}{p}}
\end{aligned}
$$

where again $\kappa_{2}(\varepsilon) \rightarrow 0$ as $\varepsilon \rightarrow 0$.

Next, we should estimate

$$
\begin{aligned}
& \left|\int_{S_{\varepsilon}} g_{z}^{\prime}\left(\frac{x}{\varepsilon}, u_{\delta}\right)\left(\left(u_{\varepsilon}-\bar{u}_{\varepsilon}\right)-u_{\varepsilon}^{\delta}\right) \mathrm{d} \mathcal{H}^{n-1}\right|=\left|\int_{S_{\varepsilon}} g_{z}^{\prime}\left(\frac{x}{\varepsilon}, u_{\delta}\right)\left(\left(u_{\varepsilon}-u_{\varepsilon}^{\delta}\right)-\overline{\left(u_{\varepsilon}-u_{\varepsilon}^{\delta}\right)}\right) \mathrm{d} \mathcal{H}^{n-1}\right| \leq \\
& c \int_{S_{\varepsilon}}\left|\left(u_{\varepsilon}-u_{\varepsilon}^{\delta}\right)-\overline{\left(u_{\varepsilon}-u_{\varepsilon}^{\delta}\right)}\right| \mathrm{d} \mathcal{H}^{n-1} \leq c\left(\int_{\Omega_{\varepsilon}}\left|D\left(u_{\varepsilon}-u_{\varepsilon}^{\delta}\right)\right|^{p} \mathrm{~d} x\right)^{1 / p} \\
& =c\left(\int_{\Omega_{\varepsilon}}\left|D\left(\left(1-\varphi_{\varepsilon}^{\delta}\right)\left(u_{\varepsilon}-u\right)\right)\right|^{p} \mathrm{~d} x\right)^{1 / p} \leq\left(\int_{A_{\varepsilon}^{\delta}}\left|D\left(u_{\varepsilon}-u\right)\right|^{p} \mathrm{~d} x\right)^{1 / p}+\frac{1}{\delta^{2}}\left(\int_{A_{\varepsilon}^{\delta}}\left|u_{\varepsilon}-u\right|^{p} \mathrm{~d} x\right)^{1 / p} \\
& \leq c \delta^{1 / p}+\frac{c}{\delta^{2}} \kappa_{3}(\varepsilon)
\end{aligned}
$$

with $\kappa_{3}(\varepsilon) \rightarrow 0$ as $\varepsilon \rightarrow 0$; the bound (4.4) and the estimate $\left|D \varphi_{\varepsilon}^{\delta}\right| \leq c / \delta^{2}$ have also been used here.

Combining the above estimates we obtain (4.7). 
Step 5. Denote by $\mathcal{C}$ the discontinuity set of $D u$. Since $u$ is a piecewise affine function, then $\mathcal{C}$ consists of finite number of hyperplanes. For every $\delta>0$, let us consider the set $B_{\delta}=\cup\left\{Y_{\delta}^{\alpha}: Y_{\delta}^{\alpha} \cap \mathcal{C} \neq \emptyset\right.$ or $\left.Y_{\delta}^{\alpha} \cap \partial \Omega \neq \emptyset\right\}$. It is easy to see that $\left|B_{\delta}\right| \leq c \delta$. Under our standing assumptions,

$$
F_{\varepsilon}\left(u_{\varepsilon}, B_{\delta}\right) \geq-c \delta^{\frac{n p-n+p}{n p}} \wedge 1, \quad\left|F\left(u, B_{\delta}\right)\right| \leq c_{1} \delta .
$$

Indeed, by Lemma 2.1

$$
F_{\varepsilon}\left(u_{\varepsilon}, B_{\delta}\right) \geq-c \int_{B_{\delta}}\left(1+\left|u_{\varepsilon}\right|^{p}\right) \geq-c\left(\left|B_{\delta}\right|^{\frac{n p-n+p}{n p} \wedge 1}+\left|B_{\delta}\right|\right) ;
$$

the last inequality here follows from the bound $\left\|u_{\varepsilon}\right\|_{W^{1, p}(\Omega)} \leq C$ and the Sobolev imbedding theorem. The second inequality in (4.8) is trivial.

Taking into account the definitions of $L(z, \xi)$ and of $u_{\varepsilon}^{\delta}$ (see (4.5)), we obtain

$$
\int_{\Omega_{\varepsilon} \backslash B_{\delta}} f\left(\frac{x}{\varepsilon}, D u_{\varepsilon}^{\delta}\right) \mathrm{d} x+\int_{S_{\varepsilon} \backslash B_{\delta}} g_{z}^{\prime}\left(\frac{x}{\varepsilon}, u_{\delta}\right)\left(u_{\varepsilon}^{\delta}-u_{\delta}\right) \mathrm{d} \mathcal{H}^{n-1} \geq \int_{\Omega_{\backslash B_{\delta}}} L\left(u_{\delta}, D u\right) \mathrm{d} x .
$$

In order to prove this inequality we denote $\mathcal{I}_{\delta}=\left\{\alpha \in \mathbb{Z}^{n}: Y_{\delta}^{\alpha} \subset \Omega \backslash B_{\delta}\right\}$, and $\mathcal{J}_{\varepsilon}=\left\{i: Y_{\varepsilon}^{i} \subset \Omega \backslash B_{\delta}\right\}$. Then

$$
\begin{aligned}
\int_{\Omega_{\varepsilon} \backslash B_{\delta}} f\left(\frac{x}{\varepsilon}, D u_{\varepsilon}^{\delta}\right) \mathrm{d} x+ & \int_{S_{\varepsilon} \backslash B_{\delta}} g_{z}^{\prime}\left(\frac{x}{\varepsilon}, u_{\delta}\right)\left(u_{\varepsilon}^{\delta}-u_{\delta}\right) \mathrm{d} \mathcal{H}^{n-1} \geq \\
& \sum_{\alpha \in \mathcal{I}_{\delta}} \inf _{w \in W_{\text {per }}^{1, p}\left(Y_{\delta}^{\alpha}\right)} \int_{Y_{\delta}^{\alpha} \cap \Omega_{\varepsilon}} f\left(\frac{x}{\varepsilon}, D u+D w\right) \mathrm{d} x+\int_{Y_{\delta}^{\alpha} \cap S_{\varepsilon}} g_{z}^{\prime}\left(\frac{x}{\varepsilon}, u_{\delta}\right)(u+w) \mathrm{d} \mathcal{H}^{n-1} \\
= & \sum_{i \in \mathcal{J}_{\varepsilon}} \inf _{w \in W_{\mathrm{Per}}^{1, p}\left(Y_{\varepsilon}^{i}\right)} \int_{Y_{\varepsilon}^{i} \cap \Omega_{\varepsilon}} f\left(\frac{x}{\varepsilon}, D u+D w\right) \mathrm{d} x+\int_{Y_{\varepsilon}^{i} \cap S_{\varepsilon}} g_{z}^{\prime}\left(\frac{x}{\varepsilon}, u_{\delta}\right)(u+w) \mathrm{d} \mathcal{H}^{n-1} \\
= & \int_{\Omega_{\backslash} \backslash B_{\delta}} L\left(u_{\delta}, D u\right) \mathrm{d} x
\end{aligned}
$$

the first equality here follows from the convexity of the Lagrangian with respect to the function $w$ and its gradient.

Step 6. Considering the properties of $L(z, \xi)$ it is easy to see that

$$
\left|\int_{\Omega \backslash B_{\delta}} L\left(u_{\delta}, D u\right) \mathrm{d} x-\int_{\Omega \backslash B_{\delta}} L(u, D u) \mathrm{d} x\right| \leq c \delta
$$

as $\delta \rightarrow 0$.

The last two inequalities together with the estimates (4.6), (4.7) and (4.8) imply

$$
\liminf _{\varepsilon \rightarrow 0} F_{\varepsilon}\left(u_{\varepsilon}\right) \geq \int_{\Omega} L(u, D u) \mathrm{d} x-c\left(\delta+\delta^{1 / p}+\delta^{\frac{n p-n+p}{n p} \wedge 1}\right) .
$$

Since $\delta$ is an arbitrary positive number, the desired $\Gamma$-lim inf inequality (4.1) follows.

\section{Proof of Theorem 2.3: the $\Gamma$-lim sup inequality}

According to the definition of $\Gamma$-convergence, we have to prove that for every $u \in W^{1, p}(\Omega)$ there exists a sequence $u_{\varepsilon} \in W^{1, p}(\Omega) \rightarrow u$ strongly in $L^{p}(\Omega)$, such that

$$
\limsup _{\varepsilon \rightarrow 0} F_{\varepsilon}\left(u_{\varepsilon}\right) \leq F(u)
$$


Since the functional $F$ is continuous with respect to strong convergence in $W^{1, p}(\Omega)$, it is enough to show that (5.1) holds for every piecewise affine function $u$. Moreover, since

$$
F(u)=\int_{\Omega} L(u, D u) \mathrm{d} x
$$

then, by localization, we can reduce to the case where $u$ is affine, i.e., $u(x)=\hat{u}+\xi \cdot x$, where $\hat{u} \in \mathbb{R}$ and $\xi \in \mathbb{R}^{n}$.

First of all, given $z \in \mathbb{R}$, and $\xi \in \mathbb{R}^{n}$, we fix a solution $w(\cdot, z, \xi) \in W_{\text {per }}^{1, p}(Y)$ of the minimum problem (1.14) such that

$$
\bar{w}=\mathcal{H}^{n-1}(S \cap Y)^{-1} \int_{S \cap Y} w \mathrm{~d} \mathcal{H}^{n-1}=0 .
$$

For every $\delta>0$, with $0<\varepsilon<\delta<1$, let us define by $Q^{\delta}$ the open cube $\left.Q^{\delta}=\right]-\frac{\delta}{2}, \frac{\delta}{2}\left[{ }^{n}\right.$, and by $Q^{\delta+}$ and $Q^{\delta-}$ the sightly bigger and smaller ones $\left.Q^{\delta+}=\right]-\frac{\delta+\delta^{2}}{2}, \frac{\delta+\delta^{2}}{2}\left[{ }^{n}, Q^{\delta-}=\right]-\frac{\delta-\delta^{2}}{2}, \frac{\delta-\delta^{2}}{2}\left[{ }^{n}\right.$. For every $x_{j}^{\delta} \in \delta \mathbb{Z}^{n}$, we denote their translated images by $Q_{j}^{\delta}, Q_{j}^{\delta+}, Q_{j}^{\delta-}$, i.e. $Q_{j}^{\delta}+x_{j}^{\delta}, Q_{j}^{\delta+}+x_{j}^{\delta}, Q_{j}^{\delta-}+x_{j}^{\delta}$. Let $J_{\delta}=\left\{j \in \mathbb{Z}^{n}: Q_{j}^{\delta+} \cap \bar{\Omega} \neq \emptyset\right\}$. To the family of cubes $\left(Q_{j}^{\delta+}\right)$, with $j \in J_{\delta}$, we can associate a finite partition of unity on the set $\bar{\Omega}$, by choosing $\Phi_{j}^{\delta} \in \mathcal{C}_{0}^{\infty}\left(Q_{j}^{\delta+}\right)$, such that $0 \leq \Phi_{j}^{\delta} \leq 1, \Phi_{j}^{\delta} \equiv 1$ on $Q_{j}^{\delta-},\left|D \Phi_{j}^{\delta}\right| \leq \frac{c}{\delta^{2}}$ with fixed $c>0$, and $\sum_{j \in J_{\delta}} \Phi_{j}^{\delta}=1$ on $\bar{\Omega}$.

For every $\varepsilon, \delta>0$ and every $j \in J$ let us define the $\varepsilon$-periodic function

$$
w_{\varepsilon j}^{\delta}(x)=w\left(\frac{x}{\varepsilon}, \hat{u}+\xi \cdot x_{j}^{\delta}, \xi\right), \quad \forall x \in \mathbb{R}^{n} .
$$

Set also

$$
v_{\varepsilon}^{\delta}(x)=\sum_{j \in J} \Phi_{j}^{\delta}(x) w_{\varepsilon j}^{\delta}(x) \quad \forall x \in \Omega
$$

and

By construction and by (3.14)

$$
u_{\varepsilon}^{\delta}=\hat{u}+\xi \cdot x+\varepsilon v_{\varepsilon}^{\delta} .
$$

$$
\left\|v_{\varepsilon}^{\delta}\right\|_{L^{p}(\Omega)} \leq c \quad \forall \varepsilon, \delta
$$

and hence

$$
\left\|u_{\varepsilon}^{\delta}-(\hat{u}+\xi \cdot x)\right\|_{L^{p}(\Omega)} \leq c \varepsilon \quad \forall \varepsilon, \delta .
$$

Moreover, from (3.15) we also have

$$
\left\|D u_{\varepsilon}^{\delta}\right\|_{L^{p}(\Omega)} \leq c\left(1+\frac{\varepsilon}{\delta^{2}}\right) \quad \forall \varepsilon, \delta .
$$

Now we claim that, provided $\delta$ is an integer multiple of $\varepsilon$, we have

$$
F_{\varepsilon}\left(u_{\varepsilon}^{\delta}\right) \leq \sum_{j \in J_{\delta}} L\left(\hat{u}+\xi \cdot x_{j}^{\delta}, \xi\right)\left|Q_{j}^{\delta}\right|+k(\varepsilon, \delta),
$$

where

$$
k(\varepsilon, \delta) \leq c\left(\varepsilon+\delta+\frac{\varepsilon}{\delta^{2}}\right)+c \max \left\{\varepsilon, \varepsilon^{p-1}\right\}\left(1+\left(\frac{\varepsilon}{\delta^{2}}\right)^{p}\right)
$$

Note that, for a proper choice of $\delta=\delta(\varepsilon), \lim _{\varepsilon \rightarrow 0} k(\varepsilon, \delta(\varepsilon))=0$. Hence, from (5.7), by choosing $\delta=\delta(\varepsilon) \rightarrow 0$ and $u_{\varepsilon}=u_{\varepsilon}^{\delta} \rightarrow u$, we will obtain finally that

$$
\limsup _{\varepsilon \rightarrow 0} F_{\varepsilon}\left(u_{\varepsilon}\right) \leq \limsup _{\delta \rightarrow 0} \sum_{j \in J_{\delta}} L\left(\hat{u}+\xi \cdot x_{j}^{\delta}, \xi\right)\left|Q_{j}^{\delta}\right|=\int_{\Omega} L(u, D u) \mathrm{d} x=F(u)
$$


The the inequality (5.7) will be derived through several steps. We recall that

$$
F_{\varepsilon}\left(u_{\varepsilon}^{\delta}\right)=\int_{\Omega_{\varepsilon}} f\left(\frac{x}{\varepsilon}, D u_{\varepsilon}^{\delta}\right) \mathrm{d} x+\int_{S_{\varepsilon}} g\left(\frac{x}{\varepsilon}, u_{\varepsilon}^{\delta}\right) \mathrm{d} \mathcal{H}^{n-1},
$$

and $D u_{\varepsilon}^{\delta}=\xi+\varepsilon D v_{\varepsilon}^{\delta}$.

Step 1. We start by estimating the bulk energy. We want to show that there is a constant $c>0$ such that

$$
\int_{\Omega_{\varepsilon}} f\left(\frac{x}{\varepsilon}, \xi+\varepsilon D v_{\varepsilon}^{\delta}\right) \mathrm{d} x \leq \sum_{j \in J} \int_{Q_{j}^{\delta} \cap \Omega_{\varepsilon}} f\left(\frac{x}{\varepsilon}, \xi+D_{y} w\left(\frac{x}{\varepsilon}, \hat{u}+\xi \cdot x_{j}^{\delta}, \xi\right)\right) \mathrm{d} x+c \varepsilon\left(1+\frac{1}{\delta^{2}}\right)+c \delta
$$

for all $\varepsilon$ and $\delta$, such that $0<\varepsilon<\delta<1$. Since

$$
D u_{\varepsilon}^{\delta}=\xi+\varepsilon D v_{\varepsilon}^{\delta}=\xi+\varepsilon \sum_{j \in J} w_{\varepsilon j}^{\delta} D \Phi_{j}^{\delta}+\sum_{j \in J} \Phi_{j}^{\delta} D_{y} w_{\varepsilon j}^{\delta},
$$

we can rewrite the volume integral as follows

$$
\begin{aligned}
\int_{\Omega_{\varepsilon}} f\left(\frac{x}{\varepsilon}, D u_{\varepsilon}^{\delta}\right) \mathrm{d} x= & \int_{\Omega_{\varepsilon}} f\left(\frac{x}{\varepsilon}, \xi+\sum_{j \in J} \Phi_{j}^{\delta} D_{y} w_{\varepsilon j}^{\delta}\right) \mathrm{d} x \\
& +\left[\int_{\Omega_{\varepsilon}} f\left(\frac{x}{\varepsilon}, D u_{\varepsilon}^{\delta}\right) \mathrm{d} x-\int_{\Omega_{\varepsilon}} f\left(\frac{x}{\varepsilon}, \xi+\sum_{j \in J} \Phi_{j}^{\delta} D_{y} w_{\varepsilon j}^{\delta}\right) \mathrm{d} x\right]
\end{aligned}
$$

Due to the convexity of $f(y, \xi)$ with respect to $\xi$, we have

$$
\int_{\Omega_{\varepsilon}} f\left(\frac{x}{\varepsilon}, \xi+\sum_{j \in J} \Phi_{j}^{\delta} D_{y} w_{\varepsilon j}^{\delta}\right) \mathrm{d} x \leq \sum_{j \in J_{\delta}} \int_{\Omega_{\varepsilon} \cap Q_{j}^{\delta+}} f\left(\frac{x}{\varepsilon}, \xi+D_{y} w_{\varepsilon j}^{\delta}\right) \mathrm{d} x
$$

Moreover, since

$$
|f(y, \xi)-f(y, \eta)| \leq c\left(1+|\xi|^{p-1}+|\eta|^{p-1}\right)|\xi-\eta|, \quad \forall \xi, \eta \in \mathbb{R}^{n},
$$

we can estimate the difference in square brackets in (5.10) as follows

$$
\begin{aligned}
& \left|\int_{\Omega_{\varepsilon}} f\left(\frac{x}{\varepsilon}, D u_{\varepsilon}^{\delta}\right) \mathrm{d} x-\int_{\Omega_{\varepsilon}} f\left(\frac{x}{\varepsilon}, \xi+\sum_{j \in J_{\delta}} \Phi_{j}^{\delta} D_{y} w_{\varepsilon j}^{\delta}\right) \mathrm{d} x\right| \leq \\
& \quad c \varepsilon \int_{\Omega_{\varepsilon}}\left(1+\left|D u_{\varepsilon}^{\delta}\right|^{p-1}+\left|\xi+\sum_{j \in J_{\delta}} \Phi_{j}^{\delta} D_{y} w_{\varepsilon j}^{\delta}\right|^{p-1}\right)\left|\sum_{j \in J_{\delta}} w_{\varepsilon j}^{\delta} D \Phi_{j}^{\delta}\right| \mathrm{d} x \stackrel{\text { def }}{=} J_{1}^{\varepsilon, \delta} .
\end{aligned}
$$

Due to Holder's and Young's inequalities, the last integral admits the estimate

$$
\begin{aligned}
J_{1}^{\varepsilon, \delta} & \leq c \varepsilon\left(\int_{\Omega_{\varepsilon}}\left(1+\left|D u_{\varepsilon}^{\delta}\right|^{p}+\left|\xi+\sum_{j \in J_{\delta}} \Phi_{j}^{\delta} D_{y} w_{\varepsilon j}^{\delta}\right|^{p}\right) \mathrm{d} x\right)^{\frac{p-1}{p}}\left(\int_{\Omega_{\varepsilon}}\left|\sum_{j \in J_{\delta}} w_{\varepsilon j}^{\delta} D \Phi_{j}^{\delta}\right|^{p} \mathrm{~d} x\right)^{\frac{1}{p}} \\
& \leq c \varepsilon \int_{\Omega_{\varepsilon}}\left(1+\left|D u_{\varepsilon}^{\delta}\right|^{p}+\left|\xi+\sum_{j \in J_{\delta}} \Phi_{j}^{\delta} D_{y} w_{\varepsilon j}^{\delta}\right|^{p}\right) \mathrm{d} x+c \varepsilon \int_{\Omega_{\varepsilon}}\left|\sum_{j \in J_{\delta}} w_{\varepsilon j}^{\delta} D \Phi_{j}^{\delta}\right|^{p} \mathrm{~d} x
\end{aligned}
$$


Notice that, by construction, $\left|D \Phi_{j}^{\delta}\right| \leq \frac{1}{\delta^{2}}$, and that by (3.14)

$$
\left\|w_{\varepsilon j}^{\delta}\right\|_{L^{p}\left(\Omega_{\varepsilon}\right)} \leq c\left(1+|\hat{u}|^{p}+\left.\xi\right|^{p}\right), \quad\left\|D w_{\varepsilon j}^{\delta}\right\|_{L^{p}\left(\Omega_{\varepsilon}\right)} \leq c\left(1+|\hat{u}|^{p}+\left.\xi\right|^{p}\right)
$$

for all $\varepsilon, \delta$ and $j$. Therefore,

$$
c \varepsilon \int_{\Omega_{\varepsilon}}\left(1+\left|D u_{\varepsilon}^{\delta}\right|^{p}+\left|\xi+\sum_{j \in J_{\delta}} \Phi_{j}^{\delta} D_{y} w_{\varepsilon j}^{\delta}\right|^{p}\right) \mathrm{d} x+c \varepsilon \int_{\Omega_{\varepsilon}}\left|\sum_{j \in J_{\delta}} w_{\varepsilon j}^{\delta} D \Phi_{j}^{\delta}\right|^{p} \mathrm{~d} x \leq c \varepsilon\left(1+\frac{1}{\delta^{2}}\right)
$$

for all $\varepsilon, \delta$. We have obtained the estimate

$\int_{\Omega_{\varepsilon}} f\left(\frac{x}{\varepsilon}, \xi+\varepsilon D v_{\varepsilon}^{\delta}\right) \mathrm{d} x \leq \sum_{j \in J} \int_{Q_{j}^{\delta+} \cap \Omega_{\varepsilon}} f\left(\frac{x}{\varepsilon}, \xi+D_{y} w\left(\frac{x}{\varepsilon}, \hat{u}+\xi \cdot x_{j}^{\delta}, \xi\right)\right) \mathrm{d} x+c \varepsilon\left(1+\frac{1}{\delta^{2}}\right)$.

To complete the proof of (5.9) it is sufficient to show that

$$
\left|\sum_{j \in J} \int_{\left(Q_{j}^{\delta+} \backslash Q_{j}^{\delta}\right) \cap \Omega_{\varepsilon}} f\left(\frac{x}{\varepsilon}, \xi+D_{y} w\left(\frac{x}{\varepsilon}, \hat{u}+\xi \cdot x_{j}^{\delta}, \xi\right)\right) \mathrm{d} x\right| \leq c \delta .
$$

Provided we choose $\delta^{2}$ to be an integer multiple of $\varepsilon$, the above inequality follows easily from the $\varepsilon$ periodicity of the integrand, and the fact that Lebesgue measure of the set

$$
\cup_{j}\left(Q_{j}^{\delta+} \backslash Q_{j}^{\delta}\right)
$$

can be estimated by $c \delta$.

Step 2. Now we estimate the surface integral on the right hand side of (5.8). We claim that

$$
\begin{aligned}
\int_{S_{\varepsilon}} g\left(\frac{x}{\varepsilon}, u_{\varepsilon}^{\delta}\right) \mathrm{d} \mathcal{H}^{n-1} \leq & \int_{S_{\varepsilon}} g_{z}^{\prime}\left(\frac{x}{\varepsilon}, \hat{u}+\langle\xi \cdot x\rangle\right)\left(u_{\varepsilon}^{\delta}-\overline{u_{\varepsilon}^{\delta}}\right) \mathrm{d} \mathcal{H}^{n-1} \\
& +c \max \left\{\varepsilon, \varepsilon^{p-1}\right\}\left(1+\left(\frac{\varepsilon}{\delta^{2}}\right)^{p}\right)+c(\varepsilon+\delta)
\end{aligned}
$$

where, for every function $v$, the symbol $\bar{v}$ denotes the piecewise-constant function which coincides with the integral average of $v$ on each $\varepsilon$-cell $Y_{\varepsilon}^{i}$, while $\langle\xi \cdot x\rangle$ is just defined by

$$
\langle\xi \cdot x\rangle=\sum_{j \in J_{\delta}} \chi_{Q_{j}^{\delta}} \xi \cdot x_{j}^{\delta}
$$

The bound (5.13) will be obtained through the following intermediate steps.

Step 2a. By Lemma 3.5, considering the fact that $u_{\varepsilon}^{\delta}$ satisfies estimate (5.5) for all $\varepsilon$ and $\delta$, we deduce that

$$
\int_{S_{\varepsilon}} g\left(\frac{x}{\varepsilon}, u_{\varepsilon}^{\delta}\right) \mathrm{d} \mathcal{H}^{n-1} \leq \int_{S_{\varepsilon}} g_{z}^{\prime}\left(\frac{x}{\varepsilon}, \overline{u_{\varepsilon}^{\delta}}\right)\left(u_{\varepsilon}^{\delta}-\overline{u_{\varepsilon}^{\delta}}\right) \mathrm{d} \mathcal{H}^{n-1}+c \max \left\{\varepsilon, \varepsilon^{p-1}\right\}\left(1+\frac{\varepsilon}{\delta^{2}}\right)
$$

for all $\varepsilon, \delta$.

Step 2b. Let us recall that $u_{\varepsilon}^{\delta}=\hat{u}+\xi \cdot x+\varepsilon v_{\varepsilon}^{\delta}$. Our next aim is to prove the inequality

$$
\int_{S_{\varepsilon}} g_{z}^{\prime}\left(\frac{x}{\varepsilon}, \overline{u_{\varepsilon}^{\delta}}\right)\left(u_{\varepsilon}^{\delta}-\overline{u_{\varepsilon}^{\delta}}\right) \mathrm{d} \mathcal{H}^{n-1} \leq \int_{S_{\varepsilon}} g_{z}^{\prime}\left(\frac{x}{\varepsilon}, \hat{u}+\langle\xi \cdot x\rangle\right)\left(u_{\varepsilon}^{\delta}-\overline{u_{\varepsilon}^{\delta}}\right) \mathrm{d} \mathcal{H}^{n-1}+c(\varepsilon+\delta) .
$$


The proof relies on the Lipschitz-continuity of $g_{z}^{\prime}(y, \cdot)$ (see (1.7)), and on the estimates of $L^{p}$-norms of the functions $v_{\varepsilon}^{\delta}$ over the surfaces $S_{\varepsilon}$. It is convenient to rearrange the left-hand side of (5.15) as follows

$$
\begin{aligned}
& \int_{S_{\varepsilon}} g_{z}^{\prime}\left(\frac{x}{\varepsilon}, \overline{u_{\varepsilon}^{\delta}}\right)\left(u_{\varepsilon}^{\delta}-\overline{u_{\varepsilon}^{\delta}}\right) \mathrm{d} \mathcal{H}^{n-1}=\int_{S_{\varepsilon}} g_{z}^{\prime}\left(\frac{x}{\varepsilon}, \hat{u}+\langle\xi \cdot x\rangle\right)\left(u_{\varepsilon}^{\delta}-\overline{u_{\varepsilon}^{\delta}}\right) \mathrm{d} \mathcal{H}^{n-1} \\
& \quad+\left[\int_{S_{\varepsilon}} g_{z}^{\prime}\left(\frac{x}{\varepsilon}, \overline{u_{\varepsilon}^{\delta}}\right)\left(u_{\varepsilon}^{\delta}-\overline{u_{\varepsilon}^{\delta}}\right) \mathrm{d} \mathcal{H}^{n-1}-\int_{S_{\varepsilon}} g_{z}^{\prime}\left(\frac{x}{\varepsilon}, \hat{u}+\langle\xi \cdot x\rangle\right)\left(u_{\varepsilon}^{\delta}-\overline{u_{\varepsilon}^{\delta}}\right) \mathrm{d} \mathcal{H}^{n-1}\right] .
\end{aligned}
$$

Denote the term in the square brackets here by $J_{2}^{\varepsilon, \delta}$. Then by (1.7)

$$
J_{2}^{\varepsilon, \delta} \leq c \int_{S_{\varepsilon}}\left(1+\left|\overline{u_{\varepsilon}^{\delta}}\right|+|\hat{u}+\langle\xi \cdot x\rangle|\right)^{p-2}\left|u_{\varepsilon}^{\delta}-\hat{u}-\langle\xi \cdot x\rangle\right|\left|u_{\varepsilon}^{\delta}-\overline{u_{\varepsilon}^{\delta}}\right| \mathrm{d} \mathcal{H}^{n-1} .
$$

Note that

$$
u_{\varepsilon}^{\delta}-\overline{u_{\varepsilon}^{\delta}}=\xi(x-\bar{x})+\varepsilon\left(v_{\varepsilon}^{\delta}-\overline{v_{\varepsilon}^{\delta}}\right),
$$

and that, by construction, $|x-\bar{x}| \leq \varepsilon$. Hence,

$$
\left|u_{\varepsilon}^{\delta}-\overline{u_{\varepsilon}^{\delta}}\right| \leq \varepsilon\left(|\xi|+\left|\left(v_{\varepsilon}^{\delta}-\overline{v_{\varepsilon}^{\delta}}\right)\right|\right) .
$$

Taking this estimate into account and substituting

$$
\overline{u_{\varepsilon}^{\delta}}=\hat{u}+\xi \cdot \bar{x}+\varepsilon \overline{v_{\varepsilon}^{\delta}}
$$

we obtain that

$$
J_{2}^{\varepsilon, \delta} \leq \varepsilon \int_{S_{\varepsilon}}\left(1+|\hat{u}|+|\xi|+\varepsilon\left|\overline{v_{\varepsilon}^{\delta}}\right|\right)^{p-2}\left(\left|\xi \cdot \bar{x}-\langle\xi \cdot x\rangle+\varepsilon \overline{v_{\varepsilon}^{\delta}}\right|\right)\left(|\xi|+\left|v_{\varepsilon}^{\delta}-\overline{v_{\varepsilon}^{\delta}}\right|\right) \mathrm{d} \mathcal{H}^{n-1}
$$

Applying Holder's inequality to the three terms of the integrand with the exponents $\frac{p}{p-2}, p$ and $p$, respectively, and distributing the factor $\varepsilon$ among these terms, we get

$$
\begin{aligned}
J_{2}^{\varepsilon, \delta} \leq & \left(\int_{S_{\varepsilon}} \varepsilon\left(1+|\hat{u}|+|\xi|+\varepsilon\left|\overline{v_{\varepsilon}^{\delta}}\right|\right)^{p} \mathrm{~d} \mathcal{H}^{n-1}\right)^{\frac{p-2}{p}} \\
& \times\left(\int_{S_{\varepsilon}} \varepsilon\left|\xi \cdot \bar{x}-\langle\xi \cdot x\rangle+\varepsilon \overline{v_{\varepsilon}^{\delta}}\right|^{p} \mathrm{~d} \mathcal{H}^{n-1}\right)^{\frac{1}{p}}\left(\int_{S_{\varepsilon}} \varepsilon\left(|\xi|+\left|v_{\varepsilon}^{\delta}-\overline{v_{\varepsilon}^{\delta}}\right|\right)^{p} \mathrm{~d} \mathcal{H}^{n-1}\right)^{\frac{1}{p}} .
\end{aligned}
$$

The first and third terms of this product are bounded, while the second one is not greater than $c(\varepsilon+\delta)$. In order to prove this we should estimate the $L^{p}$-norm of $v_{\varepsilon}^{\delta}$ on $S_{\varepsilon}$. To this end we recall that, by definition (5.2), at each point $x \in \Omega$ the function $v_{\varepsilon}^{\delta}(x)$ is a finite combination of the local minimizers $w_{\varepsilon j}^{\delta}$ with coefficients $\Phi_{j}^{\delta}$, and the number of terms involved is at most $2 n$. Moreover, by (3.6),

$$
\int_{S_{\varepsilon}}\left|w_{\varepsilon j}^{\delta}\right|^{p} \mathrm{~d} \mathcal{H}^{n-1} \leq c\left(\varepsilon^{-1} \int_{\Omega_{\varepsilon}}\left|w_{\varepsilon j}^{\delta}\right|^{p} \mathrm{~d} x+\varepsilon^{p-1} \int_{\Omega_{\varepsilon}}\left|D w_{\varepsilon j}^{\delta}\right|^{p} \mathrm{~d} x\right)
$$

for every $j \in J$ and $\varepsilon, \delta>0$. Since $w_{\varepsilon j}^{\delta}$ is an $\varepsilon$-periodic function, it is easy to see that

$$
\int_{\Omega_{\varepsilon}}\left|w_{\varepsilon j}^{\delta}\right|^{p} \mathrm{~d} x \leq c, \quad \int_{\Omega_{\varepsilon}}\left|D w_{\varepsilon j}^{\delta}\right|^{p} \mathrm{~d} x \leq c \varepsilon^{-p} .
$$


The last two estimates imply the bound

$$
\int_{S_{\varepsilon}}\left|w_{\varepsilon j}^{\delta}\right|^{p} \mathrm{~d} \mathcal{H}^{n-1} \leq c \varepsilon^{-1}
$$

for every $j \in J$ and $\varepsilon, \delta>0$. By Jensen's inequality we then get

$$
\int_{S_{\varepsilon}}\left|\overline{v_{\varepsilon}^{\delta}}\right|^{p} \mathrm{~d} \mathcal{H}^{n-1} \leq \int_{S_{\varepsilon}}\left|v_{\varepsilon}^{\delta}\right|^{p} \mathrm{~d} \mathcal{H}^{n-1} \leq c \varepsilon^{-1} \quad \forall \varepsilon, \delta>0 .
$$

The first integral on the right-hand side of (5.16) is bounded for by (3.6), (5.5) and (5.6) we have

$$
\begin{gathered}
\int_{S_{\varepsilon}} \varepsilon\left(1+\left|u_{\varepsilon}^{\delta}\right|+|\hat{u}+\langle\xi \cdot x\rangle|\right)^{p} \mathrm{~d} \mathcal{H}^{n-1} \leq c\left(1+\varepsilon \int_{S_{\varepsilon}}\left|u_{\varepsilon}^{\delta}\right|^{p} \mathrm{~d} \mathcal{H}^{n-1}\right) \leq \\
\left(1+\int_{\Omega_{\varepsilon}}\left|u_{\varepsilon}^{\delta}\right|^{p} \mathrm{~d} x+\varepsilon^{p} \int_{\Omega_{\varepsilon}}\left|D u_{\varepsilon}^{\delta}\right|^{p} \mathrm{~d} x\right) \leq c\left(1+\varepsilon^{p}+\varepsilon^{p}\left(\frac{\varepsilon}{\delta^{2}}\right)^{p}\right) .
\end{gathered}
$$

The second integral vanishes, as $\varepsilon, \delta \rightarrow 0$, since $|\xi \cdot \bar{x}-\langle\xi \cdot x\rangle| \leq c(\varepsilon+\delta)^{p}$ and, by (5.17), we have

$$
\int_{S_{\varepsilon}} \varepsilon\left|\xi \cdot \bar{x}-\langle\xi \cdot x\rangle+\varepsilon \overline{v_{\varepsilon}^{\delta}}\right|^{p} \mathrm{~d} \mathcal{H}^{n-1} \leq c(\varepsilon+\delta)+c \varepsilon^{p+1} \int_{S_{\varepsilon}}\left|v_{\varepsilon}^{\delta}\right|^{p} \mathrm{~d} \mathcal{H}^{n-1} \leq c\left(\delta^{p}+\varepsilon^{p}\right) .
$$

Finally, the third integral is bounded for all $\varepsilon>0$, since

$$
\varepsilon \int_{S_{\varepsilon}}\left(|\xi|+\left|v_{\varepsilon}^{\delta}-\overline{v_{\varepsilon}^{\delta}}\right|\right)^{p} \mathrm{~d} \mathcal{H}^{n-1} \leq c(\xi)\left(1+\varepsilon^{p+1} \int_{S_{\varepsilon}}\left|v_{\varepsilon}^{\delta}\right|^{p}\right) \mathrm{d} \mathcal{H}^{n-1} \leq c(\xi)\left(1+\varepsilon^{p}\right) .
$$

Combining the above bounds, we obtain (5.15).

Step 2c. To complete the proof of (5.13) it remains to show that

$$
\int_{S_{\varepsilon}} g_{z}^{\prime}\left(\frac{x}{\varepsilon}, \hat{u}+\langle\xi \cdot x\rangle\right)\left(u_{\varepsilon}^{\delta}-\overline{u_{\varepsilon}^{\delta}}\right) \mathrm{d} \mathcal{H}^{n-1}=\int_{S_{\varepsilon}} g_{z}^{\prime}\left(\frac{x}{\varepsilon}, \hat{u}+\langle\xi \cdot x\rangle\right)\left(\xi \cdot x-\langle\xi \cdot x\rangle+\varepsilon v_{\varepsilon}^{\delta}\right) \mathrm{d} \mathcal{H}^{n-1} .
$$

To this end, notice that the difference between $u_{\varepsilon}^{\delta}-\overline{u_{\varepsilon}^{\delta}}$ and $\left(\xi \cdot(x-\bar{x})+\varepsilon\left(v_{\varepsilon}^{\delta}-\overline{v_{\varepsilon}^{\delta}}\right)\right)$ is equal to a constant on each $\varepsilon$-cell provided that $\delta$ is an integer multiplier of $\varepsilon$. Then the desired relation (5.18) follows from (1.5).

Step 3. With the help of (5.13) one can prove that

$$
\begin{aligned}
\int_{S_{\varepsilon}} g_{z}^{\prime} & \left(\frac{x}{\varepsilon}, \hat{u}+\langle\xi \cdot x\rangle\right)\left(\xi \cdot x-\langle\xi \cdot x\rangle+\varepsilon v_{\varepsilon}^{\delta}\right) \mathrm{d} \mathcal{H}^{n-1} \leq \\
& \sum_{j \in J_{\delta}} \int_{Q_{j}^{\delta} \cap S_{\varepsilon}} g_{z}^{\prime}\left(\frac{x}{\varepsilon}, \hat{u}+\langle\xi \cdot x\rangle\right)\left(\xi \cdot x-\xi \cdot x_{j}^{\delta}+\varepsilon w_{\varepsilon j}^{\delta}\right) \mathrm{d} \mathcal{H}^{n-1}+c\left(\delta+\frac{\varepsilon}{\delta^{2}}\right) .
\end{aligned}
$$

Indeed, by the definition of $v_{\varepsilon}^{\delta}$ we have

$$
\begin{aligned}
\int_{S_{\varepsilon}} g_{z}^{\prime}\left(\frac{x}{\varepsilon}, \hat{u}+\langle\xi \cdot x\rangle\right)\left(\xi \cdot(x-\langle x\rangle)+\varepsilon v_{\varepsilon}^{\delta}\right) \mathrm{d} \mathcal{H}^{n-1}= \\
\sum_{j \in J_{\delta}} \int_{Q_{j}^{\delta} \cap S_{\varepsilon}} g_{z}^{\prime}\left(\frac{x}{\varepsilon}, \hat{u}+\xi \cdot x_{j}^{\delta}\right)\left(\xi \cdot x-\xi \cdot x_{j}^{\delta}+\varepsilon \Phi_{j}^{\delta} w_{\varepsilon j}^{\delta}\right) \mathrm{d} \mathcal{H}^{n-1} .
\end{aligned}
$$


Therefore, it suffices to estimate the difference

$$
J_{4}^{\varepsilon, \delta}=\left|\sum_{j \in J_{\delta}} \int_{Q_{j}^{\delta} \cap S_{\varepsilon}} g_{z}^{\prime}\left(\frac{x}{\varepsilon}, \hat{u}+\xi \cdot x_{j}^{\delta}\right) \varepsilon\left(\Phi_{j}^{\delta}-1\right) w_{\varepsilon j}^{\delta} \mathrm{d} \mathcal{H}^{n-1}\right|
$$

Recall that $\Phi_{j}^{\delta}=1$ in $Q_{j}^{\delta-}$ for any $j \in \mathbb{Z}^{n}$. If we denote $G_{j}^{\delta}=Q_{j}^{\delta} \backslash Q_{j}^{\delta-}$ and $G_{\delta}=\bigcup_{j} G_{j}^{\delta}$, then

$$
J_{4}^{\varepsilon, \delta} \leq\left|\sum_{j \in J_{\delta}} \int_{G_{j}^{\delta} \cap S_{\varepsilon}} g_{z}^{\prime}\left(\frac{x}{\varepsilon}, \hat{u}+\xi \cdot x_{j}^{\delta}\right) \varepsilon\left(\Phi_{j}^{\delta}-1\right) w_{\varepsilon j}^{\delta} \mathrm{d} \mathcal{H}^{n-1}\right|
$$

Note that $\left|\Phi_{j}^{\delta}-1\right| \leq 1$ and, by (1.4)-(1.7),

$$
\left|g_{z}^{\prime}\left(\frac{x}{\varepsilon}, \hat{u}+\langle\xi \cdot x\rangle\right)\right| \leq c \quad \text { for a.e. } x \in \Omega
$$

so that

$$
J_{4}^{\varepsilon, \delta} \leq c \sum_{j \in J_{\delta}} \int_{G_{j}^{\delta} \cap S_{\varepsilon}} \varepsilon\left|w_{\varepsilon j}^{\delta}\right| \mathrm{d} \mathcal{H}^{n-1} .
$$

Now, by the $\varepsilon$-periodicity of $w_{\varepsilon j}^{\delta}$ and $(3.14)$, for each $j$ we have

$$
\int_{G_{j}^{\delta} \cap S_{\varepsilon}} \varepsilon\left|w_{\varepsilon j}^{\delta}\right| \mathrm{d} \mathcal{H}^{n-1}=\varepsilon^{n-1} \int_{S \cap Y} \varepsilon\left|w\left(y, \hat{u}+\xi \cdot x_{j}^{\delta}, \xi\right)\right| \mathrm{d} \mathcal{H}^{n-1} \cdot\left|G_{j}^{\delta}\right| \varepsilon^{-n} \leq c \delta^{n+1}
$$

a simple bound $\left|G_{j}^{\delta}\right| \leq c \delta^{n+1}$ has also been used here. Since the cardinality of $J_{\delta}$ is of the order $\delta^{-n}$, then, summing up over $j$ and considering the fact that the cardinality of $J_{\delta}$ is not greater than bound $c \delta^{-n}$, we conclude that

$$
\sum_{j \in J_{\delta}} \int_{G_{j}^{\delta} \cap S_{\varepsilon}} \varepsilon\left|w_{\varepsilon j}^{\delta}\right| \mathrm{d} \mathcal{H}^{n-1} \leq c \delta
$$

which completes the proof of (5.19).

Step 4. Taking into account the estimates of Steps $1-3$, we arrive at the inequality

$$
\begin{aligned}
F_{\varepsilon}\left(u_{\varepsilon}^{\delta}\right) \leq & \sum_{j \in J} \int_{Q_{j}^{\delta} \cap \Omega_{\varepsilon}} f\left(\frac{x}{\varepsilon}, \xi+D_{y} w\left(\frac{x}{\varepsilon}, \hat{u}+\xi \cdot x_{j}^{\delta}, \xi\right)\right) \mathrm{d} x \\
& +\sum_{j \in J} \int_{Q_{j}^{\delta} \cap S_{\varepsilon}} g_{z}^{\prime}\left(\frac{x}{\varepsilon}, \hat{u}+\xi \cdot x_{j}^{\delta}\right)\left(\xi \cdot x-\xi \cdot x_{j}^{\delta}+\varepsilon w_{\varepsilon j}^{\delta}\right)+k(\varepsilon, \delta)
\end{aligned}
$$

with

$$
k(\varepsilon, \delta)=c\left(\varepsilon+\delta+\frac{\varepsilon}{\delta^{2}}\right)+c \max \left\{\varepsilon, \varepsilon^{p-1}\right\}\left(1+\left(\frac{\varepsilon}{\delta^{2}}\right)^{p}\right)
$$


In order to prove (5.7) it is sufficient to observe that

$$
\begin{gathered}
\sum_{j \in J_{\delta}} \int_{Q_{j}^{\delta} \cap \Omega_{\varepsilon}} f\left(\frac{x}{\varepsilon}, \xi+D_{y} w\left(\frac{x}{\varepsilon}, \hat{u}+\xi \cdot x_{j}^{\delta}, \xi\right)\right) \mathrm{d} x+\sum_{j \in J_{\delta}} \int_{Q_{j}^{\delta} \cap S_{\varepsilon}} g_{z}^{\prime}\left(\frac{x}{\varepsilon}, \hat{u}+\xi \cdot x_{j}^{\delta}\right)\left(\xi \cdot x-\xi \cdot x_{j}^{\delta}+\varepsilon w_{\varepsilon j}^{\delta}\right)= \\
\sum_{j \in J_{\delta}} L\left(\hat{u}+\xi \cdot x_{j}^{\delta} \cdot \xi\right)\left|Q_{j}^{\delta}\right| .
\end{gathered}
$$

The $\Gamma$-limsup inequality is proved.

Proof of Proposition 2.4. For an arbitrary $u \in W^{1, p}(\Omega),\left.u\right|_{\partial \Omega}=\Phi$, we are going to modify the family $u_{\varepsilon}$ so that for the modified functions the relation (2.4) still holds true and they satisfy the boundary condition $\left.u_{\varepsilon}\right|_{\partial \Omega}=\Phi$. Clearly, it suffices to show that for any $\delta>0$ there is a sequence $v_{\varepsilon}^{\delta} \in W^{1, p}(\Omega)$ such that $v_{\varepsilon}^{\delta} \rightarrow u$ in $W^{1, p}(\Omega)$ as $\varepsilon \rightarrow 0,\left.v_{\varepsilon}^{\delta}\right|_{\partial \Omega}=\Phi$ and

where $\kappa_{5}(\delta) \rightarrow 0$ as $\delta \rightarrow 0$.

$$
\limsup _{\varepsilon \rightarrow 0} F^{\varepsilon}\left(v_{\varepsilon}^{\delta}\right) \leq F(u)+\kappa_{5}(\delta),
$$

Let $u^{\delta}$ be a piece-wise affine function in $\mathbb{R}^{n}$ such that $\left\|u-u^{\delta}\right\|_{W^{1, p}(\Omega)} \leq \delta$. As was shown in the beginning of this section, there is a family $\left\{u_{\varepsilon}^{\delta}\right\}$ (see (5.2), (5.3)) such that

$$
\limsup _{\varepsilon \rightarrow 0} F^{\varepsilon}\left(u_{\varepsilon}^{\delta}\right)=F\left(u^{\delta}\right) .
$$

Denote by $L_{\Omega}^{\nu}$ the $\nu$-neighbourhood of $\partial \Omega$ intersected with $\Omega$. By construction,

$$
\limsup _{\varepsilon \rightarrow 0}\left\|u_{\varepsilon}^{\delta}\right\|_{W^{1, p}\left(L_{\Omega}^{\nu}\right)} \rightarrow 0
$$

as $\nu \rightarrow 0$. Let $\phi_{\nu}(x)$ be a cut-off function such that $\phi_{\nu} \in C_{0}^{\infty}(\Omega), 0 \leq \phi_{\nu} \leq 1, \phi_{\nu}(x)=1$ if $\operatorname{dist}(x, \partial \Omega)>\nu$, and $\left|\nabla \phi_{\nu}\right| \leq 2 / \nu$. If we set $u_{\varepsilon}^{\delta, \nu}=u^{\delta}+\left(u_{\varepsilon}^{\delta}-u^{\delta}\right) \phi_{\nu}$, then $u_{\varepsilon}^{\delta, \nu}=u^{\delta}$ on $\partial \Omega$, and

$$
\begin{gathered}
\left\|u_{\varepsilon}^{\delta, \nu}-u_{\varepsilon}^{\delta}\right\|_{L^{p}(\Omega)} \leq C\left(\left\|u^{\delta}\right\|_{L^{p}\left(L_{\Omega}^{\nu}\right)}+\left\|u_{\varepsilon}^{\delta}\right\|_{L^{p}\left(L_{\Omega}^{\nu}\right)}\right), \\
\left\|D u_{\varepsilon}^{\delta, \nu}-D u_{\varepsilon}^{\delta}\right\|_{L^{p}(\Omega)} \leq C\left(\left\|D u^{\delta}\right\|_{L^{p}\left(L_{\Omega}^{\nu}\right)}+\left\|D u_{\varepsilon}^{\delta}\right\|_{L^{p}\left(L_{\Omega}^{\nu}\right)}+\frac{1}{\nu}\left\|u_{\varepsilon}^{\delta}-u^{\delta}\right\|_{L^{p}(\Omega)}\right) .
\end{gathered}
$$

The last inequalities yield

$$
\limsup _{\varepsilon \rightarrow 0}\left\|u_{\varepsilon}^{\delta, \nu}-u_{\varepsilon}^{\delta}\right\|_{W^{1, p}(\Omega)} \rightarrow 0,
$$

as $\nu \rightarrow 0$. Choosing now $\nu=\nu(\delta)$ in such a way that

$$
\limsup _{\varepsilon \rightarrow 0}\left\|u_{\varepsilon}^{\delta, \nu}-u_{\varepsilon}^{\delta}\right\|_{W^{1, p}(\Omega)} \leq \delta
$$

and letting $v_{\varepsilon}^{\delta}=u_{\varepsilon}^{\delta, \nu}+\left(u-u^{\delta}\right)$, by Lemma 3.6 we obtain the desired inequality (5.20).

\section{REFERENCES}

[1] E. Acerbi, V. Chiadò Piat, G. Dal Maso and D. Percivale, An extension theorem from connected sets, and homogenization in general periodic domains. Nonlinear Anal. 18 (1992) 481-496.

[2] R.A. Adams, Sobolev spaces. Academic Press, New York (1975).

[3] A.G. Belyaev, G.A. Chechkin and A.L. Piatnitski, Asymptotic behavior of a solution to a boundary value problem in a perforated domain with oscillating boundary. Sib. Math. J. 39 (1998) 621-644.

[4] A.G. Belyaev, G.A. Chechkin and A.L. Piatnitski, Homogenization of second-order elliptic operators in a perforated domain with oscillating Fourier boundary conditions. Sb. Math. 192 (2001) 933-949. 
[5] A. Braides and A. Defranceschi, Homogenization of multiple integrals, Oxford Lecture Series in Mathematics and its Applications 12. The Clarendon Press, Oxford University Press, New York (1998).

[6] A. Brillard, Asymptotic analysis of two elliptic equations with oscillating terms. RAIRO Modél. Math. Anal. Numér. 22 (1988) 187-216.

[7] D. Cioranescu and P. Donato, On a Robin problem in perforated domains, in Homogenization and applications to material sciences, D. Cioranescu et al. Eds., GAKUTO International Series, Mathematical Sciences and Applications 9, Tokyo, Gakkotosho (1997) 123-135.

[8] D. Cioranescu and F. Murat, A strange term coming from nowhere, in Topics in the Mathematical Modelling of Composite Materials, Progr. Nonlinear Differential Equations Appl. 31, Birkhauser, Boston (1997) 45-93.

[9] D. Cioranescu and J. Saint Jean Paulin, Homogenization in open sets with holes. J. Math. Anal. Appl. 71 (1979) 590-607.

[10] D. Cioranescu and J. Saint Jean Paulin, Truss structures: Fourier conditions and eigenvalue problems, in Boundary control and boundary variation, J.P. Zolezio Ed., Lecture Notes Control Inf. Sci. 178, Springer-Verlag (1992) 125-141.

[11] C. Conca, On the application of the homogenization theory to a class of problems arising in fluid mechanics. J. Math. Pures Appl. 64 (1985) 31-75.

[12] G. Dal Maso, An introduction to $\Gamma$-convergence. Birkhauser, Boston (1993).

[13] V.A. Marchenko and E.Y. Khruslov, Boundary value problems in domains with fine-grained boundaries. Naukova Dumka, Kiev (1974).

[14] V.A. Marchenko and E.Y. Khruslov, Homogenization of partial differential equations. Birkhauser (2006).

[15] O.A. Oleinik and T.A. Shaposhnikova, On an averaging problem in a partially punctured domain with a boundary condition of mixed type on the boundary of the holes, containing a small parameter. Differ. Uravn. 31 (1995) 1150-1160, 1268. Translation in Differ. Equ. 31 (1995) 1086-1098.

[16] O.A. Oleinik and T.A. Shaposhnikova, On the homogenization of the Poisson equation in partially perforated domains with arbitrary density of cavities and mixed type conditions on their boundary. Rend. Mat. Acc. Linceis. IX 7 (1996) 129-146.

[17] S.E. Pastukhova, Tartar's compensated compactness method in the averaging of the spectrum of a mixed problem for an elliptic equation in a punctured domain with a third boundary condition. Sb. Math. 186 (1995) 753-770.

[18] S.E. Pastukhova, On the character of the distribution of the temperature field in a perforated body with a given value on the outer boundary under heat exchange conditions on the boundary of the cavities that are in accord with Newton's law. Sb. Math. 187 (1996) 869-880.

[19] S.E. Pastukhova, Spectral asymptotics for a stationary heat conduction problem in a perforated domain. Mat. Zametki 69 (2001) 600-612 [in Russian]. Translation in Math. Notes 69 (2001) 546-558.

[20] W.P. Ziemer, Weakly differentiable functions. Springer-Verlag, New York (1989). 